\title{
The $S p_{3}$-grassmannian and duality for prime Fano threefolds of genus 9
}

\author{
Atanas Iliev $\mathrm{G}$
}

\begin{abstract}
By a result of Mukai, the non-abelian Brill-Noether locus $X=M_{C}(2, K: 3 F)$ of type II, defined by a stable rank 2 vector bundle $F$ of invariant 3 over a plane quartic curve $C$, is a prime Fano 3-fold $X=X_{16}$ of degree 16 . The associate ruled surface $S^{X}=\mathbf{P}(F)$ is uniquely defined by $X$, and we see that for the general $X=X_{16}, S^{X}$ is isomorphic to the Fano surface of conics on $X$. The argument uses the geometry of the $S p_{3}$-grassmannian and the double projection from a line on $X_{16}$.
\end{abstract}

\section{$\S 1$. Introduction}

The smooth Fano 3-fold $X$ is prime if $\operatorname{Pic}(X)=\mathbf{Z}\left(-K_{X}\right)$, where $K_{X}$ is the canonical class of $X$. The degree $d=d(X)=-K_{X}{ }^{3}=2 g(X)-2$ of a prime Fano 3-fold $X$ is always even, and the integer $g=g(X)$ is called the genus of $X=X_{2 g-2}$. Prime Fano 3-folds $X_{2 g-2}$ exist iff $2 \leq g=g(X) \leq 12, g \neq 11$, see [I], [IP].

Let $C$ be a smooth plane quartic curve, and let $S=\mathbf{P}(F) \rightarrow C$ be a stable ruled surface of invariant $e(S)=\min \left\{s^{2}: s\right.$ a section of $\left.S\right\}=3$. By a result of Mukai the non-abelian Brill-Noether locus of type II

$$
X_{F}=M_{C}(2, K: 3 F)=\{E \rightarrow C: \operatorname{rank} E=2, \operatorname{det} E=\operatorname{det} F \otimes K, \operatorname{dim} H o m(F, E) \geq 3\},
$$

in the moduli space $M_{C}\left(2\right.$, det $\left.F \otimes K_{C}\right)$ of stable rank 2 vector bundles on $C$ of determinant $\operatorname{det} F \otimes K_{C}$, is a prime Fano threefold of genus 9 , see [M3]. The associate Fano 3 -fold $X_{S}:=X_{F}$ is uniquely defined by $S$, since the locus $X_{F}=X_{F \otimes L}$ does not depend on the twists $F \mapsto F \otimes L$ by line bundles $L$ on $C$, see [M3]. In turn, the general Fano 3-fold $X$ of genus 9 is associate to a unique ruled surface $S=S^{X}$, over the associate plane quartic curve $C^{X}$ of $X$, see (5.7).

By [M1], M2], any prime Fano 3 -fold $X=X_{16}$ of genus 9 is a linear section of the $S p_{3^{-}}$ grassmannian $\Sigma \subset \mathbf{P}^{13}$ by a codimension 3 subspace $\mathbf{P}^{10}=\mathbf{P}_{X}^{10} \subset \mathbf{P}^{13}$, defined uniquely by $X$ upto the action $\rho$ of the symplectic group $S p_{3}$ on $\mathbf{P}^{13}$.

The dual projective representation $\hat{\rho}$ of $\rho$ has an invariant quartic hypersurface $\hat{F}$ in the dual space $\hat{\mathbf{P}}^{13}$, see (2.3)(ii), (2.5.a). For a given general $X=\Sigma \cap \mathbf{P}_{X}^{10}$, the plane $\mathbf{P}_{X}^{2}=\left(\mathbf{P}_{X}^{10}\right)^{\perp} \subset \hat{\mathbf{P}}^{13}$ intersects $\hat{F}$ along a smooth plane quartic curve $C_{X}$, defined uniquely by $X$, see (4.1).

The two main results in this paper are:

\footnotetext{
*Partially supported by Grant MM-1106/2001 of the Bulgarian Foundation for Scientific Research
} 
A. The Fano family $\mathcal{F}(X)$ of conics on the general prime Fano 3 -fold $X$ of genus 9 is a ruled surface over the $S p_{3}$-dual plane quartic $C_{X}$ of $X$ : see Theorem (4.6).

B. The ruled surface $\mathcal{F}(X)$ is isomorphic to the associate ruled surface $S^{X}$ of $X$; in particular $C_{X} \cong C^{X}$ : see Theorem (6.5).

The proof of $\mathbf{A}$ uses the geometry of $X$ as a subvariety of the $S p_{3}$-grassmannian $\Sigma \subset \mathbf{P}^{13}$. The points $c$ of the dual quartic curve $C_{X}$ of $X$ are the singular hyperplane sections $H_{c} \subset \Sigma$ which contain $X$. For any $c \in C_{X}$, the hyperplane section $H_{c}$ has a node at the correlative pivot point $\hat{u}(c) \in \Sigma$ of $c$, see (2.7) and (4.1). In the lagrangian plane $\mathbf{P}_{\hat{u}(c)}^{2}$ lies a smooth conic $q(c)$, invariant under the action of the stabilizer subgroup $S t_{\hat{c}} \subset S p_{3}$ of $H_{c}$, see (3.3). The vertex surface $S_{X}=\cup_{c \in C_{X}} q(c) \subset \mathbf{P}^{5}$ of $X$ is a ruled surface over the dual curve $C_{X}$ with fibers the smooth conics $q(c)$. The points $x \in S_{X}$ are the vertices of the conics $q_{x} \subset X$ (see (4.4)), and the vertex map $q_{x} \mapsto x$ sends the Fano surface $\mathcal{F}(X)$ of conics on $X$ isomorphically onto the ruled vertex surface $S_{X}$ of $X$, see (4.5.b-d) and (4.6). This proves $\mathbf{A}$.

The proof of $\mathbf{B}$ is based on the simultaneous interpretation of the lines $l=l_{\varepsilon}=l_{L}$ on the Fano threefold $X$ as minimal sections $C_{\varepsilon}$ of the associate surface $S^{X}$ and as sections $C_{L}$ of the vertex surface $S_{X}$. By [[स], a minimal section $C_{\varepsilon} \subset S^{X}$ is represented by a space curve $C_{\varepsilon}^{l}$ of genus 3 and degree 7 . By $\mathrm{M} 3$ the curve $C_{\varepsilon}^{l}$ is the same as the curve $C^{l}$ defining the inverse to a double projection from the line $l=l_{\varepsilon}$ on $X$, see (5.1) and (5.4)(d). As a line on the $S p_{3}$-grassmannian, $l=l_{L}$ represents a pencil of lagrangian planes through an isotropic line $L$, in a 3-space $\mathbf{P}_{L}^{3} \subset \mathbf{P}^{5}$, see (3.1). In the intersection of $\mathbf{P}_{L}^{3}$ with the vertex surface $S_{X}$ lies a unique curve $C_{L}$, which is a section of the ruled vertex surface $S_{X}$. By the $S p_{3}$-geometry of the double projection from $l \subset X \subset \Sigma$, the curve $C_{L}$ is projectively equivalent to $C^{l}$, see (6.1)-(6.4). The correspondence $C_{\varepsilon} \leftrightarrow l_{\varepsilon}=l=l_{L} \leftrightarrow C_{L}$ identifies the set $\operatorname{Min}\left(S^{X}\right)$ of minimal sections $C_{\varepsilon}$ of $S^{X}$ and the set $\operatorname{Min}^{\prime}\left(S_{X}\right)$ of sections $C_{L}$ of $S_{X}$. At the end, Theorem (6.5) shows that the isomorphism $\operatorname{Min}\left(S^{X}\right) \cong \operatorname{Min}^{\prime}\left(S_{X}\right)$ yields an isomorphism between the associate surface $S^{X}$ and the isomorphic vertex model $S_{X}$ of $\mathcal{F}(X)$. This proves B.

\section{§. The Sp3-grassmannian $\Sigma \subset \mathbf{P}^{13}$}

(2.1) Let $V_{6}=\mathbf{C}^{6}$ be a complex vector 6 -space, let $\hat{V}_{6}=H o m\left(V_{6}, \mathbf{C}\right)$ be its dual space, and fix a rank six 2-form $\alpha \in \wedge^{2} \hat{V}_{6}$. The 21-dimensional symplectic group $S p_{3}=S p_{3}^{\alpha} \subset G L_{6}$ is the set of all $A \in G L_{6}$ which preserve the non-degenerate skew-symmetric product $\alpha: V_{6} \times V_{6} \rightarrow \mathbf{C}$ defined by $\alpha$. In coordinates $\left(e_{i}, x_{i}\right)=\left(e_{1}, \ldots e_{6} ; x_{1}, \ldots, x_{6}\right)$ on $V_{6}$

$$
S p_{3}=\left\{A \in G L_{6}:{ }^{t} A J A=J\right\}
$$

where $J_{i j}=\alpha\left(e_{i}, e_{j}\right)$ is the skew-symmetric Gramm matrix of $\alpha$. One can always choose the coordinates $\left(e_{i}, x_{i}\right)$ on $V_{6}$ such that $\alpha=x_{14}+x_{25}+x_{36}$; and then $J_{i j}=J_{3+i, 3+j}=0$, $J_{i, 3+j}=\delta_{i j}=-J_{3+i, j}, 1 \leq i, j \leq 3$.

The subspace $U \subset V_{6}$ is called isotropic if $\left.\alpha\right|_{U} \equiv 0$, i.e. $\alpha\left(u_{1}, u_{2}\right)=0$ for any $u_{1}, u_{2} \in U$; and then the projective subspace $\mathbf{P}(U) \subset \mathbf{P}\left(V_{6}\right)=\mathbf{P}^{5}$ is also called isotropic.

Since $\alpha$ is skew-symmetric then any point $x \in \mathbf{P}^{5}$ is isotropic. Since $\alpha$ is non-degenerate then the dimension of an isotropic $U \subset V$ can't be more than 3. The isotropic subspaces $U \subset V_{6}$ of dimension 3, as well their projective planes $\mathbf{P}(U) \subset \mathbf{P}^{5}$, are called lagrangian. 
The isotropic grassmannian $L G_{2} \subset G\left(2, V_{6}\right)$ is the set of all isotropic lines $L \subset \mathbf{P}^{5}$. One can see that the line $L=\mathbf{P}\left(U_{2}\right)$ is isotropic iff its Plücker image $\mathbf{P}\left(\wedge^{2} U_{2}\right)$ lies in the isotropic hyperplane $(\alpha=0) \subset \mathbf{P}\left(\wedge^{2} V_{6}\right)$, i.e.

$$
L G_{2}=G(2,6) \cap(\alpha=0)=G(2,6) \cap\left(x_{14}+x_{25}+x_{36}=0\right) ;
$$

and since $\alpha$ is non-degenerate, $L G_{2}$ is a smooth hyperplane section of $G(2,6)$.

The lagrangian grassmannian, or the $S p_{3}$-grassmannian $\Sigma=L G_{3} \subset G\left(3, V_{6}\right)$ is the set of all lagrangian subspaces $U \subset V_{6}$. The $S p_{3}$-grassmannian, as well the isotropic grassmannian $L G_{2}$, is a smooth homogeneous variety of the group $S p_{3}$. In the paper we shall use the explicit coordinate description of $\Sigma$ which we state below.

For the 3-space $U \subset V_{6}$, the condition $\left.\alpha\right|_{U} \equiv 0$ is equivalent to the requirement that its Plücker image $p l(U)=\mathbf{P}\left(\wedge^{3} U\right) \in \mathbf{P}^{19}=\mathbf{P}\left(\wedge^{3} V_{6}\right)$ lies in the codimension 6 subspace $\mathbf{P}^{13}=$ $\mathbf{P}\left(V_{14}\right)=\left(\hat{V}_{6} \wedge \alpha=0\right) \subset \mathbf{P}^{19}$, i.e.

$$
\Sigma=G(3,6) \cap\left(\hat{V}_{6} \wedge \alpha=0\right)=G(3,6) \cap\left(x_{i 14}+x_{i 25}+x_{i 36}=0,1 \leq i \leq 6\right) .
$$

Let $U_{o}=<e_{1}, e_{2}, e_{3}>$ and $U_{\infty}=<e_{4}, e_{5}, e_{6}>$. Then $V_{6}=U_{o} \oplus U_{\infty}$, and in homogeneous coordinates

$(u: X: Y: z)=\left(u:\left(x_{i j}\right):\left(y_{i j}\right): z\right):=\left(x_{123}:\left(\begin{array}{lll}x_{423} & x_{143} & x_{124} \\ x_{523} & x_{153} & x_{125} \\ x_{623} & x_{163} & x_{126}\end{array}\right):\left(\begin{array}{lll}x_{156} & x_{416} & x_{451} \\ x_{256} & x_{426} & x_{452} \\ x_{356} & x_{436} & x_{453}\end{array}\right): x_{456}\right)$

on $\mathbf{P}^{19}=\mathbf{P}\left(\wedge^{3}\left(U_{o} \oplus U_{\infty}\right)\right)=\mathbf{P}\left(\wedge^{3} U_{o} \oplus \wedge^{2} U_{o} \otimes U_{\infty} \oplus U_{o} \otimes \wedge^{2} U_{\infty} \oplus \wedge^{3} U_{\infty}\right)$, the equations $\hat{V}_{6} \wedge \alpha=0$ of $\mathbf{P}^{13} \subset \mathbf{P}^{19}(u: X: Y: z)$ become ${ }^{t} X=X,{ }^{t} Y=Y$; i.e.

$$
\mathbf{P}^{13}=\mathbf{P}^{13}(u: X: Y: z),{ }^{t} X=X,{ }^{t} Y=Y .
$$

Let $G(3,6)^{o}=G(3,6)-H_{o}^{G}$ be the complement to the Schubert hyperplane section $H_{o}^{G}=$ $\sigma_{100}\left(U_{o}\right)=\left\{U \in G(3,6): \operatorname{dim}\left(U \cap U_{o}\right)>0\right\}=G(3,6) \cap(u=0)$. Let also $H_{o} \subset \Sigma$ be the hyperplane section $\Sigma \cap H_{o}^{G}=\Sigma \cap(u=0)$, and $\Sigma^{o}=\Sigma-H_{o}$. The 3-spaces $U=U_{X} \in G(3,6)^{o}$ are parameterized $1: 1$ by the linear maps $X_{U}: U_{o} \rightarrow U_{\infty}$, by

$$
U=<e_{1}+X\left(e_{1}\right), e_{2}+X\left(e_{2}\right), e_{3}+X\left(e_{3}\right)>\longmapsto X \in \operatorname{Hom}\left(U_{o}, U_{\infty}\right) \cong \otimes^{2} \mathbf{C}^{3},
$$

and then $p l(U)=\left(1: X: \wedge^{2} X: \operatorname{det} X\right)$. Moreover, the 3 -space $U=U_{X} \in G(3,6)^{\circ}$ is lagrangian (i.e. $U \in \Sigma^{o}$ ) iff the matrix $X=X_{U}$ is symmetric. Therefore $\Sigma$ is the natural projective compactification of the isomorphic image $\Sigma^{o}=\Sigma \cap(u \neq 0)$ of the affine space $\mathbf{C}^{6}=$ Sym $^{2} \mathbf{C}^{3}$ under the exp-map:

$$
\exp : \operatorname{Sym}^{2} \mathbf{C}^{3} \rightarrow \mathbf{P}^{13}(u: X: Y: z), \quad X \mapsto \exp (X)=\left(1: X: \wedge^{2} X: \operatorname{det} X\right) .
$$

As a subvariety of $\mathbf{P}^{13}(u: X: Y: z),{ }^{t} X=X,{ }^{t} Y=Y$, the smooth 6 -fold $\Sigma$ is defined by the 21 projectivized quadratic Cramer equations

$$
\wedge^{2} X=u Y, \wedge^{2} Y=z X, X Y=u z I_{3}
$$

(where $I_{3}$ is the unit $3 \times 3$ matrix), which follow from the local exp-parameterization of $\Sigma$ by the skew-powers of symmetric $3 \times 3$ matrices. 
(2.2) Remark. The next Theorem (2.3) and Lemma (2.7) below are particular cases of the more general Propositions 5.10-5.11 and Proposition 8.2 in [LM1, where are described the orbits in the enveloping spaces and the singular hyperplane sections of the four varieties $\Sigma_{A}=$ $G_{\omega}\left(A^{3}, A^{6}\right)$, in the third row of the Freudenthal magic square. The four varieties $G_{\omega}\left(A^{3}, A^{6}\right)$ correspond to the four complex composition algebras $A=\mathbf{A}_{\mathbf{R}} \times \mathbf{C}$, where $\mathbf{A}_{\mathbf{R}}=\mathbf{R}, \mathbf{C}, \mathbf{H}$ and $\mathbf{O}=$ the real numbers, the complex numbers, the quaternions and the octonions. The $S p_{3^{-}}$ grassmannian $\Sigma=\Sigma_{R}$ corresponds to the case $A=R$; see also $\S 3$ in [D], where these results are proved in case $A=C$, where $\Sigma_{C}=G(3,6)$.

The subspace $V_{14} \subset \wedge^{3} V_{6}$ is the irreducible representation space of $S p_{3}$, defined by the weight $\wedge_{3}$, see [FH], p. 258. We shall denote by $\rho$ the induced projective action of $\wedge_{3}$ on $\mathbf{P}^{13}=\mathbf{P}\left(V_{14}\right)$.

(2.3) Theorem of Segre for $\mathbf{S p}_{3}$. The action $\rho: S p_{3} \times \mathbf{P}^{13} \rightarrow \mathbf{P}^{13}$ has 4 orbits

$$
\mathbf{P}^{13}=\Sigma \cup(\Omega-\Sigma) \cup(F-\Omega) \cup\left(\mathbf{P}^{13}-F\right), \quad \text { and }:
$$

(i) $\Sigma$ is the isomorphic Plücker image of the $S p_{3}$-grassmannian $L G_{3}$.

(ii) $F \subset \mathbf{P}^{13}$ is a quartic hypersurface. Moreover $F=\cup_{u \in \Sigma} \mathbf{P}_{u}^{6}$, where $\mathbf{P}_{u}^{6}$ denotes the tangent projective 6-space at the point $u \in \Sigma$.

(iii) $x \in \mathbf{P}^{13}-\Omega \Leftrightarrow$ there exists a unique secant or tangent line $l_{x}$ to $\Sigma$, s.t. $x \in l_{x}$. Moreover $x \in F-\Omega \Leftrightarrow l_{x}$ is tangent to $\Sigma$.

For the point $u \in \Sigma$ we shall denote by $\mathbf{P}_{u}^{2} \subset \mathbf{P}^{5}$ the lagrangian plane of $u$.

(2.4) Lemma. Let $u \in \Sigma$, and let $\mathbf{P}_{u}^{6}$ be the tangent projective space of $\Sigma \subset \mathbf{P}^{13}$ at $u$. Then $\mathbf{P}_{u}^{6} \subset F$, and: (i) $K_{u}=\left\{v \in \Sigma: \operatorname{dim}\left(\mathbf{P}_{v}^{2} \cap \mathbf{P}_{u}^{2}\right) \geq 1\right\}=\Sigma \cap \mathbf{P}_{u}^{6}$ is a cone with vertex $u$ over the Veronese surface. (ii) $D_{u}=\Omega \cap \mathbf{P}_{u}^{6}$ is a cone with vertex $u$ over the symmetric determinantal cubic.

Proof. By the $S p_{3}$-homogeneity of $\Sigma$ one can let $u=(1: 0: 0: 0)$ in $\mathbf{P}^{13}(u: X: Y: z)$. In the notation of (2.1), the lagrangian plane $\mathbf{P}_{u}^{2}=\mathbf{P}\left(U_{o}\right)$, and the projective tangent space $\mathbf{P}_{u}^{6}=\mathbf{P}\left(\wedge^{3} U_{o} \oplus \wedge^{2} U_{o} \otimes U_{\infty}\right)=\mathbf{P}_{o}^{6}(u: X: 0: 0)$. Therefore $v \in \Sigma \cap \mathbf{P}^{6} \quad$ iff $\quad v \in \Sigma$ and $\operatorname{dim}\left(\mathbf{P}_{v}^{2} \cap \mathbf{P}_{u}^{2}\right) \geq 1$. By (2.1)(*), the intersection $\Sigma \cap \mathbf{P}_{u}^{6}(u: X: 0: 0)$ is defined by the system $\operatorname{rank} X=1,{ }^{t} X=X$, which implies (i). By (2.3)(iii) the intersection $\Omega \cap \mathbf{P}_{u}^{6}(u: X: 0: 0)$ is exactly the determinantal cubic cone $D_{u}=(\operatorname{det} X=0)$ which is the locus of points $\omega \in \mathbf{P}_{u}^{6}$ through which pass more than one secant line to $K_{u}$, see also $\S 9$ of [K]. q.e.d.

(2.5.a) The varieties $\boldsymbol{\Sigma} \subset \boldsymbol{\Omega} \subset \mathbf{F} \subset \mathbf{P}^{\mathbf{1 3}}$. The smooth variety $\Sigma \subset \mathbf{P}^{13}$ is a Fano 6-fold of degree 16 and of index 4, i.e. $K_{\Sigma}=\mathcal{O}_{\Sigma}(-4)$, see e.g. [M2], [M4]. In coordinates $(u: X: Y: z)$ as above, the $S p_{3}$-invariant quartic hypersurface $F$ is defined by the equation

$$
F(u: X: Y: z)=(u z-\operatorname{tr} X Y)^{2}+4 u \operatorname{det} Y+4 z \operatorname{det} X-4 \Sigma_{i j} \operatorname{det}\left(X_{i j}\right) \cdot \operatorname{det}\left(Y_{i j}\right)=0,
$$

see [KS p. 83, or $\S 5.2$ and Proposition 5.8 in [LM1. The 9 -fold $\Omega=\operatorname{Sing} F=(\nabla F=0)$ is the common zero-locus of the 14 partial cubic derivatives of $F$. It has degree 21 , and $\operatorname{Sing} \Omega=\Sigma$, see $\S 2$ of [IR]. 
(2.5.b) The pivot-map. By analogy with $\S 3$ in $[D]$, call the axis of $x \in \mathbf{P}^{13}-\Omega$ the unique secant or tangent line $l_{x}$ to $\Sigma$ through $x$, and the pivots of $x$ the two intersection points $u$ and $v$ of $l_{x}$ with $\Sigma$. If $x \in F-\Omega$ call the point $u(x)=u=v \in \Sigma$ the double pivot (or simply - the pivot) of $x$. This way, there exists a well-defined pivot-map:

$$
\text { piv }: F-\Omega \rightarrow \Sigma, x \mapsto \text { the double pivot } u=u(x) \text { of } x \text {. }
$$

The fiber of the regular pivot map piv : $F-\Omega \rightarrow \Sigma$ over the point $u \in \Sigma$ coincides with $\mathbf{P}_{u}^{6}-D_{u}$, where $D_{u}=\Omega \cap \mathbf{P}_{u}^{6}$ is the determinantal cubic cone in $\mathbf{P}_{u}^{6}$, see (2.3)-(2.4).

(2.5.c) The correlation $\mathbf{J}_{\alpha}$. The dual representation of $\wedge_{3}$ is equivalent to $\wedge_{3}$. Therefore its projective action $\hat{\rho}$ splits the dual projective space $\hat{\mathbf{P}}^{13}=\mathbf{P}\left(\hat{V}_{14}\right)$ into a union of orbits $\hat{\Sigma}$, $\hat{\Omega}-\hat{\Sigma}, \hat{F}-\hat{\Omega}$ and $\hat{\mathbf{P}}^{13}-\hat{F}$ projectively equivalent to the orbits of $\rho$. The non-degenerate form $\alpha \in \wedge^{2} \hat{V}_{6}$ defines a linear isomorphism

$$
J=J_{\alpha}: V_{6} \rightarrow \hat{V}_{6}=\operatorname{Hom}\left(V_{6}, \mathbf{C}\right), J: x \mapsto \alpha(x, .) .
$$

In canonical coordinates $\left(e_{i}, x_{i}\right)$, in which $\alpha=x_{14}+x_{25}+x_{36}$, one has: $J\left(e_{1}\right)=x_{4}$, $J\left(e_{2}\right)=x_{5}, J\left(e_{3}\right)=x_{6}, J\left(e_{4}\right)=-x_{1}, J\left(e_{5}\right)=-x_{2}$ and $J\left(e_{6}\right)=-x_{3}$.

The linear isomorphism $J$ induces a projective-linear isomorphism, or a correlation

$$
J: \mathbf{P}^{13} \rightarrow \hat{\mathbf{P}}^{13}, J: x \mapsto \hat{x}:=J(x),
$$

which identifies the elements $x$ of any of the orbits of $\rho$ with their correlative elements $J(x)=\hat{x}$ in its corresponding orbit of $\hat{\rho}$. The correlation $J$ commutes with taking pivots; in particular, for $x \in \hat{F}-\hat{\Omega}$ the correlative point $u \hat{(x)} \in \hat{\Sigma}$ of its (double) pivot $u(x)=p i v(x) \in \Sigma$ is the pivot $\hat{u}(x):=\operatorname{piv}(\hat{x})$ of its correlative point $\hat{x} \in F-\Omega$.

(2.6) Lemma. In coordinates $(u: X: Y: z)$, the correlative pivot map

$$
\widehat{p i v}: \hat{F}-\hat{\Omega} \rightarrow \Sigma, x \mapsto \widehat{u(x)},
$$

where $u(x)=\operatorname{piv}(x)$, coincides with the gradient map $\nabla=\nabla_{F}$, defined by the 14 cubic derivatives of the quartic form $F(u: X: Y: z)$.

Proof. In coordinates $(u: X: Y: z)$, let $u=(0: 0: 0: 1)=\left(x_{456}\right)$. By the $S p_{3^{-}}$ transitivity on the orbit $\hat{F}-\hat{\Omega}$, it is enough to prove that the gradient map $\nabla=\nabla_{F}$ sends any $x=(0: 0: Y: z) \in \mathbf{P}_{u}^{6}-D_{u}$ to the point $\hat{u}=\widehat{p i v(u)}=\left(\widehat{x_{456}}\right)=\left(-e_{123}\right)=\left(e_{123}\right)=(1: 0: 0: 0)$. This is direct: the straightforward check shows that

$$
\nabla_{F}:(0: 0: Y: z) \mapsto(4 \operatorname{det} Y: 0: 0: 0)=(1: 0: 0: 0)
$$

since $\operatorname{det} Y \neq 0$ on $\mathbf{P}_{u}^{6}-D_{u}$, see (2.3)-(2.4).

(2.7) Lemma. (i) If $x \in \hat{F}-\hat{\Omega}$ then the hyperplane section $H_{x} \subset \Sigma$ has a node at the correlative double pivot $\hat{u}:=u \hat{(x)}$ of $x$. Moreover $H_{x}$ contains the 3 -fold cone $K_{\hat{u}}=\mathbf{P}_{\hat{u}}^{6} \cap \Sigma$. (ii) For $u \in \hat{\Sigma}$ the hyperplane section $H_{u} \subset \Sigma$ is the same as the Schubert hyperplane section $\sigma_{100}\left(\mathbf{P}_{\hat{u}}^{2}\right) \cap \mathbf{P}^{13}=\left\{v \in \Sigma: \mathbf{P}_{v}^{2} \cap \mathbf{P}_{\hat{u}}^{2} \neq \emptyset\right\}$. The singular locus Sing $H_{u}=K_{\hat{u}}=\mathbf{P}_{\hat{u}}^{6} \cap \Sigma$ is a cone, with vertex $\hat{u}$, over the Veronese surface.

Proof: See (2.2). 


\section{$\S 3$. Lines and quadrics on the $\mathrm{Sp}_{3}$-grassmannian}

(3.1) Lemma. (i) Let $L \subset \mathbf{P}^{5}$ be an isotropic line. Then the set

$$
l_{L}=\left\{u \in \Sigma: L \subset \mathbf{P}_{u}^{2}\right\} \subset \Sigma
$$

is a line, and any line in $\Sigma$ is obtained this way.

(ii) If $\mathbf{P}_{L}^{3} \subset \mathbf{P}^{5}$ is the 3 -space such that

$$
l_{L}=\sigma_{332}\left(L, \mathbf{P}_{L}^{3}\right)=\left\{u \in \Sigma \subset G(3,6): L \subset \mathbf{P}_{u}^{2} \subset \mathbf{P}_{L}^{3}\right\}
$$

then $\mathbf{P}_{L}^{3}$ does not contain other lagrangian planes except the planes $\mathbf{P}_{u}^{2}, u \in l_{L}$.

For the line $l=l_{L}=\sigma_{322}\left(L, \mathbf{P}_{L}^{3}\right) \subset \Sigma$, we call the isotropic line $L$ and the space $\mathbf{P}_{L}^{3}$ respectively the axis and the space of $l$.

Proof. For (i), see e.g. $\S 2.7 .2$ in [LM2]. In particular, $S p_{3}$ acts transitively on the family of lines $l=l_{L} \subset \Sigma$, since the isotropic grassmannian $L G_{2}$ is a homogeneous variety of $S p_{3}$. Therefore it is enough to prove (ii) for a particular line $l$ on $\Sigma$. In coordinates $(u: X: Y: z)$, for the isotropic line $L=\mathbf{P}\left(<e_{2}, e_{3}>\right) \subset \mathbf{P}^{5}$ the space $\mathbf{P}_{L}^{3}=\mathbf{P}\left(U_{4}^{L}\right)=\mathbf{P}\left(<e_{1}, e_{2}, e_{3}, e_{4}>\right)$ and the line $l=l_{L}=\left\langle e_{123}, e_{423}>\right.$. Therefore the Plücker image $u$ of a plane $\mathbf{P}_{u}^{2} \subset \mathbf{P}_{L}^{3}$ will lie in the 3-space $\mathbf{P}_{l}^{3}=\mathbf{P}\left(\wedge^{3} U_{4}^{L}\right)=\mathbf{P}^{3}\left(x_{123}: x_{423}: x_{143}: x_{124}\right)=\mathbf{P}^{3}\left(u: x_{11}: x_{12}: x_{13}\right)$. By the equations (2.1)(*), the plane $\mathbf{P}_{u}^{2}$ will be lagrangian iff $u \in \mathbf{P}_{l}^{3} \cap\left({ }^{t} X=X,{ }^{t} Y=Y\right)=$ $\mathbf{P}^{1}\left(u: x_{11}\right)=\mathbf{P}^{1}\left(x_{123}: x_{423}\right)=l$. q.e.d.

(3.2) Lemma. Let $x \in \mathbf{P}^{5}$. Then the set

$$
Q_{x}:=\sigma_{300}(x) \cap \mathbf{P}^{13}=\left\{u \in \Sigma: x \in \mathbf{P}_{u}^{2}\right\} \subset \Sigma
$$

is a smooth 3-fold quadric, and any 3 -fold quadric on $\Sigma$ is one of the quadrics $Q_{x}, x \in \mathbf{P}^{5}$.

For the quadric $Q=Q_{x} \subset \Sigma$, we call the point $\operatorname{ver}(Q):=x \in \mathbf{P}^{5}$ the vertex of $Q$.

Proof. See $\S 2.7 .2$ in LM2]; see also the proof of (3.3)(i) below.

(3.3) Proposition : quadrics on singular hyperplane sections of $\boldsymbol{\Sigma}$.

(i) Let $Q=Q_{x} \subset \Sigma$ be a 3-fold quadric on $\Sigma$ (see (3.2)), and let $H_{c}, c \in \hat{\mathbf{P}}^{13}$ be a hyperplane section of $\Sigma$ containing $Q$. Then $c \in \hat{F}$; and if $c \notin \hat{\Omega}$, then the correlative pivot $\hat{u}(c)$ of $c$ lies on the quadric $Q$.

(ii) Let $c \in \hat{F}-\hat{\Omega}$, and let $\hat{u}=\hat{u}(c)$ be the correlative pivot of $c$. Then the set

$$
q(c)=\left\{x \in \mathbf{P}^{5}: Q=Q_{x} \subset H_{c}\right\}
$$

is a smooth conic in the lagrangian plane $\mathbf{P}_{\hat{u}}^{2}$ of $\hat{u}$, which we call the vertex conic of $c$.

Proof of (i). By the $S p_{3}$-homogeneity we can assume that $x=e_{1}$, and then in coordinates $(u: X: Y: z)=\left(u:\left(x_{i j}\right):\left(y_{i j}\right): z\right)$

$$
Q_{e_{1}}=\left(u y_{11}=x_{22} x_{23}-x_{23}^{2}\right) \subset \mathbf{P}_{e_{1}}^{4}=\mathbf{P}^{4}\left(u: x_{22}: x_{23}: x_{33}: y_{11}\right) .
$$

Any $H_{c}$ which contains $Q_{x}=Q_{e_{1}}$ contains $\mathbf{P}_{x}^{4}=\mathbf{P}_{e_{1}}^{4}$. Therefore

$$
H_{c} \supset Q_{e_{1}} \Leftrightarrow c \in \mathbf{P}_{e_{1}}^{8}:=\left(\mathbf{P}_{e_{1}}^{4}\right)^{\perp}=\left(u=x_{12}=x_{13}=x_{23}=y_{11}=0\right) \subset \hat{\mathbf{P}}^{13}(u: X: Y: z) .
$$


Now the straightforward check shows that: 1. If $c \in \mathbf{P}_{e_{1}}^{8}$ then $F(c)=0$, see (2.5.a). Therefore $c \in \hat{F}$. 2. If $c \in \mathbf{P}_{e_{1}}^{8}$ then all the partial derivatives $F_{z}(c), F_{x_{1 i}}(c), i=1,2,3$ and $F_{y_{i j}}(c),(i, j) \neq(1,1)$ vanish. Therefore $\nabla_{F}(c) \in \mathbf{P}_{e_{1}}^{4}$; and since $\nabla_{F}(c)=\hat{u}(c) \in \Sigma$ is the correlative pivot of $c$ (see (2.6)), then $\hat{u}(c) \in \Sigma \cap \mathbf{P}_{e_{1}}^{4}=Q_{e_{1}}$. q.e.d.

Proof of (ii). Let $\rho: S p_{3} \times \mathbf{P}^{13} \rightarrow \mathbf{P}^{13}, \rho_{g}: x \rightarrow \rho_{g}(x)$ be the projective action of $S p_{3}$ on $\mathbf{P}^{13}$ (see (2.2)), and let $\rho_{1}: x \mapsto g(x)$ be the projectivized standard action $\wedge_{1}$ of $S p_{3} \subset G L_{6}$ on $\mathbf{P}^{5}$. By the transitivity of $\hat{\rho}$ on the orbit $\hat{F}-\hat{\Omega}$, we can choose $c=(0: 0: Y: 0)$, where $Y$ is any symmetric rank 3 matrix (see (2.3)-(2.4)), and let $Y$ be the matrix of the quadratic form $2 y_{1} y_{2}+y_{3}^{2}$. Then $c=2 y_{12}+y_{33}=x_{416}+x_{256}+x_{453}$ : mod. $\mathbf{C}^{*}$, and the pivot $u(c)=\operatorname{piv}(c)=(0: 0: 0: 1)=\left(x_{456}\right)$. The correlative point of $c$ is $\hat{c}=\left(e_{143}+e_{523}+e_{126}\right) \in F-\Omega$ (see (2.5.c)), and the pivot of $\hat{c}$ is $\hat{u}=\operatorname{piv}(\hat{c})=(1: 0: 0: 0) \in \Sigma$, with a lagrangian plane $\mathbf{P}_{\hat{u}}^{2}=\mathbf{P}^{2}\left(x_{1}: x_{2}: x_{3}\right)=\mathbf{P}\left(U_{o}\right)$. Let

$$
P S t_{\hat{c}}=\left\{g \in S p_{3}: \rho_{g}(\hat{c})=\hat{c}\right\} / \mathbf{C}^{*} \subset P S t_{3}
$$

be the projectivized stabilizer group of $\hat{c}$.

(*) Lemma. The group $P S t_{\hat{c}} \subset$ Aut $H_{c}$; and the correlative pivot plane $\mathbf{P}_{\hat{u}}^{2}$ is invariant under the standard action $\rho_{1}$ of $P S t_{\hat{c}}$. Moreover $P S t_{\hat{c}}$ is a projectivized semi-direct product $\mathbf{P}\left(O_{q}(3) \otimes G_{a}^{5}\right)$, where $P O_{q}(3)$ is the projectivized orthogonal group of the quadric $q=q(c)=$ $\left(2 x_{1} x_{2}+x_{3}^{2}=0\right) \subset \mathbf{P}_{\hat{u}}^{2}=\mathbf{P}^{2}\left(x_{1}: x_{2}: x_{3}\right)$, and $G_{a}^{5}$ is the additive group of $\mathbf{C}^{5}$.

Proof. See $\S 9(2)$ in [K], or (5.21) in [KS].

Let $Q=Q_{x}$ be a 3-fold quadric in $H_{c}$. By (i), the vertex $x$ of $Q=Q_{x}$ lies in $\mathbf{P}_{\hat{u}}^{2}$; and by Lemma $(*)$, for any $g \in P S t_{\hat{c}}$ the quadric $\rho_{g}\left(Q_{x}\right)=Q_{g(x)}$ also lies in $H_{c}$. Therefore $Q=Q_{x}$ lies in $H_{c}$, together with all the quadrics $Q_{g(x)}, g \in P S t_{\hat{c}}$. Again by Lemma $(*)$, the action $\rho_{1}:(g, x) \mapsto g(x)$ of $P S t_{\hat{c}}$ on $\mathbf{P}_{\hat{u}}^{2}$ coincides with the action of $P O_{q}(3), q=q(c)$. Therefore $P S t_{\hat{c}}$ has two orbits in $\mathbf{P}_{\hat{u}}^{2}=\mathbf{P}^{2}\left(x_{1}: x_{2}: x_{3}\right)$ - the smooth conic $q=q(c)=\left(2 x_{1} x_{2}+x_{3}^{2}=0\right)$, and its complement $\mathbf{P}_{\hat{u}}^{2}-q(c)$.

Therefore in order to prove (ii) it is enough to see that: $\mathbf{1}$. for some $x \in q(c)$ the 5 -fold $H_{c}$ contains the quadric $Q_{x}$. 2. for some $x \in \mathbf{P}_{\hat{u}}^{2}$ the quadric $Q_{x}$ does not lie in $H_{c}$.

This is straightforward: If $x=e_{1} \in q(c)$ then the quadric $Q_{e_{1}}$ lies in $H_{c}=\Sigma \cap\left(2 y_{12}+y_{33}=0\right)$, while for the point $x=e_{3} \in \mathbf{P}_{\hat{u}}^{2}-q(c)$ the quadric $Q_{e_{3}}$ does not lie in $H_{c}$. q.e.d.

(3.4) Conics on $\boldsymbol{\Sigma}$. Call a formal conic on $\Sigma$ any plane $\mathbf{P}^{2} \subset \mathbf{P}^{13}=$ Span $\Sigma$ such that the intersection cycle $q=\mathbf{P}^{2} \cap \Sigma$ is of dimension 1 and of degree 2. Let $\mathcal{F}(\Sigma) \subset G(3,14)$ be the family of formal conics on $\Sigma$. Since $\Sigma$ is an intersection of quadrics (see (2.1) $(*)$ ), and $\Sigma$ does not contain planes (see e.g. Lemma 2.5.1 in [IR]), then the definition is correct. The rank of the formal conic $\mathbf{P}^{2}$ is the rank of the conic $q=\mathbf{P}^{2} \cap \Sigma \subset \mathbf{P}^{2}$. If $\operatorname{rank}(q)=2$ or 3 , then one can identify the conic $q \subset \Sigma$ and the formal conic $\mathbf{P}^{2}=\operatorname{Span}(q)$.

(3.5) Lemma. If $q \subset \Sigma$ is a conic of rank $\geq 2$, then there exists a unique point $x=x(q) \in$ $\mathbf{P}^{5}$, such that $q \subset Q_{x}$.

We call the point $x=x(q)$ the vertex of the rank $\geq 2$ conic $q$. 
Proof. Since $q \subset \Sigma \subset G(3,6)$ is a conic then the union $Q(q):=\cup_{u \in q} \mathbf{P}_{u}^{2}$ is either a $\mathbf{P}^{3}$ or a 3-fold quadric.

Suppose first that $Q(q)=\mathbf{P}^{3}$, i.e. all the $\mathbf{P}_{u}^{2}, u \in q$ lie in $\mathbf{P}^{3}$; and let $u, v \in q, u \neq v$. Since the lagrangian planes $\mathbf{P}_{u}^{2}$ and $\mathbf{P}_{v}^{2}$ both lie in $\mathbf{P}^{3}$, then they will intersect each other along an isotropic line $L$. By (3.1)(i), the line $l=l_{L}$ with axis $L$ contains the points $u$ and $v$, therefore $l=\operatorname{Span}(u, v)$ and $\mathbf{P}_{L}^{3}=\mathbf{P}^{3}$. But by (3.1)(ii) the only lagrangian planes in $\mathbf{P}_{L}^{3}$ are $\mathbf{P}_{w}^{2}, w \in l$. Therefore $q \subset l$ (as sets), which is only possible if $\operatorname{rank}(q)=1$ - contradiction.

Therefore $Q(q) \subset \mathbf{P}^{4}$ is a quadric, and since on $Q(q)$ lie planes, $Q(q)$ is singular.

If $q=l+m$ is of rank 2 then $Q(q)$ is the union of the spaces $\mathbf{P}_{L}^{3}$ and $\mathbf{P}_{M}^{3}$ of the lines $l$ and $m$. Let $L \subset \mathbf{P}_{L}^{3}$ and $M \subset \mathbf{P}_{M}^{3}$ be the axes of $l$ and $m$. Since $l \cap m=u$ is a point, then $\operatorname{Span}(L \cup M)=\mathbf{P}_{u}^{2}$. Therefore $L \cap M=x=x(q)$ is a point, and $q=l+m \subset Q_{x}$.

If $\operatorname{rank}(q)=3$ then $Q(q)$ is irreducible, and since on $Q(q)$ lie planes then $Q(q)$ is singular and all the $\mathbf{P}_{u}^{2}, u \in q$ pass through the subspace $\mathbf{P}^{k}=\operatorname{Sing} Q(q), k=0,1$. If $k=1$ then $\mathbf{P}^{k}=L$ is a line. The line $L$ is isotropic since it lies on lagrangian planes, and let $l=l_{L}$ be the line with axis $L$. But then $\mathbf{P}_{u}^{2} \supset L, \forall u \in q$, together with (3.1)(i), will imply $q \subset l$-contradiction. Therefore $k=0, x=x(q):=\mathbf{P}^{k}=\mathbf{P}^{0}$ is a point, and $q \subset Q_{x}$. q.e.d.

(3.6) Lemma. Let $l \subset \Sigma$ be a line. Then the set $\mathbf{P}_{l} \subset G(3,14)$ of these planes $\mathbf{P}^{2} \subset \mathbf{P}^{13}$ for which the intersection cycle $\mathbf{P}^{2} \cap \Sigma=2 . l$, is a line in $G(3,14)$.

Proof. Let $\mathbf{P}^{2} \cap \Sigma=2 . l$, and let $(u, v)$ be any pair of non-coincident points of $l$. Since $\mathbf{P}^{2} \cap \Sigma=2 . l$ then $\mathbf{P}^{2} \subset \mathbf{P}_{u}^{6} \cap \mathbf{P}_{v}^{6}$, where $\mathbf{P}_{u}^{6}$ and $\mathbf{P}_{v}^{6}$ are the tangent projective spaces to $\Sigma$ at $u$ and $v$, see (2.3)-(2.4). As in the proof of (3.1), we may assume that $l=l_{L}=$ $\operatorname{Span}(u, v)=\operatorname{Span}\left(e_{123}, e_{423}\right)$ is the line with axis $L=\operatorname{Span}\left(e_{2}, e_{3}\right)$. Therefore $\mathbf{P}_{l}^{3}=\mathbf{P}_{u}^{6} \cap$ $\mathbf{P}_{v}^{6}=\operatorname{Span}\left(e_{123}, e_{423}, e_{143}+e_{523}, e_{124}+e_{623}\right)$ is a projective 3 -space containing the line $l=$ $\operatorname{Span}\left(e_{123}, e_{423}\right)$. Now it is clear that $\mathbf{P}_{l}^{3}=\cap_{u \in l} \mathbf{P}_{u}^{6}$, and the set $\mathbf{P}_{l}$ coincides with the Schubert line $\sigma_{11,11,10}\left(l, \mathbf{P}_{l}^{3}\right)=\left\{\mathbf{P}^{2} \subset \mathbf{P}^{13}: l \subset \mathbf{P}^{2} \subset \mathbf{P}_{l}^{3}\right\} \subset G(3,14)$. q.e.d.

(3.7) Corollary. Let $\mathcal{F}_{k}(\Sigma) \subset \mathcal{F}(\Sigma) \subset G(3,14)$ be the loci of formal conics on $\Sigma$ of rank $\leq k, k=1,2$. Then $\operatorname{dim} \mathcal{F}(\Sigma)=11, \operatorname{dim} \mathcal{F}_{2}(\Sigma)=10$, and $\operatorname{dim} \mathcal{F}_{1}(\Sigma)=8$.

Proof. By (3.4) any conic $q$ of rank $>1$ can be identified with its formal conic $\mathbf{P}^{2}(q)=$ $\operatorname{Span}(q)$. By (3.5) any such $q$ is a linear section of a unique quadric $Q_{x(q)}$ with the plane $\mathbf{P}^{2}(q) \subset$ $\mathbf{P}_{x(q)}^{4}=\operatorname{Span} Q_{x(q)}$. Therefore $\operatorname{dim} \mathcal{F}(\Sigma)=\operatorname{dim} \mathbf{P}^{5}+\operatorname{dim} G(3,5)=11$, and $\operatorname{dim} \mathcal{F}_{2}(\Sigma)=10$. At the end, (3.1) and (3.6) imply: $\operatorname{dim} \mathcal{F}_{1}(\Sigma)=\operatorname{dim} L G_{2}+1=8$, see (2.1). q.e.d.

\section{$\S 4$. The Fano family $\mathcal{F}(\mathrm{X})$ of conics on $\mathrm{X}$}

(4.1) The dual plane quartic $\mathbf{C}_{\mathbf{X}}$ of $\mathbf{X}$. By [M1, [M2] any smooth prime Fano 3-fold $X=X_{16}$ of degree 16 is a linear section of the $S p_{3}$-grassmannian $\Sigma \subset \mathbf{P}^{13}$ by a codimension 3 subspace $\mathbf{P}^{10} \subset \mathbf{P}^{13}$. Moreover two prime Fano 3 -folds $X_{16}^{\prime}=\Sigma \cap \mathbf{P}_{1}^{10}$ and $X_{16}^{\prime \prime}=\Sigma \cap \mathbf{P}_{2}^{10}$ are projectively equivalent iff $\mathbf{P}_{1}^{10}$ and $\mathbf{P}_{2}^{10}$ are conjugate under the action $\rho$ of $S p_{3}$ in $\mathbf{P}^{13}$.

From now on we shall consider only the situation when $X$ is general. 
Fix a general prime Fano 3-fold $X=X_{16}=\Sigma \cap \mathbf{P}_{X}^{10}$; and let $\mathbf{P}_{X}^{2}=\mathbf{P}_{X}^{10}{ }^{\perp} \subset \hat{\mathbf{P}}^{13}$ be the plane of linear equations of $\mathbf{P}_{X}^{10} \subset \mathbf{P}^{13}$. Since $X$ is general then the plane $\mathbf{P}_{X}^{2}=\mathbf{P}_{X}^{10} \subset \hat{\mathbf{P}}^{13}$ of linear equations of $\mathbf{P}_{X}^{10} \subset \mathbf{P}^{13}$ intersects the invariant quartic hypersurface $\hat{F}$ along a smooth plane quartic curve

$$
C_{X}=\hat{F} \cap \mathbf{P}_{X}^{2}
$$

By the preceding, $C_{X}$ is uniquely defined by the Fano 3 -fold $X$; and we call the curve $C_{X} \subset \mathbf{P}_{X}^{2}$ the dual plane quartic of $X$.

Since $X$ is general then the plane $\mathbf{P}_{X}^{2}$ does not intersect the codimension 3 orbit $\hat{\Omega}$. Therefore any point $c \in C_{X}$ has a uniquely defined double pivot $u=u(c) \in \hat{\Sigma}$. By (2.7)(i)-(ii), the hyperplane sections $H_{c}$ and $H_{u}$ of $\Sigma$ both contain the Veronese cone $K_{\hat{u}}=\mathbf{P}_{\hat{u}}^{6} \cap \Sigma$. Therefore any point $c \in C_{X}$ defines uniquely a 4 -fold linear section

$$
W_{c}=H_{c} \cap H_{u(c)} \subset \Sigma ;
$$

and since $K_{\hat{u}(c)}=\operatorname{Sing} H_{u}$ (see (2.7)(ii)) then $W_{c}$ is singular along $K_{\hat{u}(c)}$.

(4.2) Lemma. Let $c \in \hat{F}-\hat{\Omega}$, and let $q(c) \subset \mathbf{P}_{\hat{u}(c)}^{2}$ be the vertex conic of $c$, see (3.3). Then $W_{c}=\cup_{x \in q(c)} Q_{x}$.

Proof. By (2.7) and (3.2)

$$
H_{u(c)}=\sigma_{100}\left(\mathbf{P}_{\hat{u}(c)}^{2}\right) \cap \mathbf{P}^{13}=\left\{w \in \Sigma: \mathbf{P}_{w}^{2} \cap \mathbf{P}_{\hat{u}(c)}^{2} \neq \emptyset\right\}=\cup_{x \in \mathbf{P}_{\hat{u}(c)}^{2}} Q_{x} .
$$

Moreover, by (3.3) any quadric $Q_{x}, x \in q(c) \subset \mathbf{P}_{\hat{u}(c)}^{2}$ lies in $H_{c}$. q.e.d.

(4.3) The singular hyperplane sections $\mathbf{S}_{\mathbf{c}} \subset \mathbf{X}$. For the general $X$, the dual curve $C_{X}$ does not intersect $\hat{\Omega}$, hence the pivot map piv : $C_{X} \rightarrow \hat{\Sigma}, c \mapsto u(c)=p i v(c)$ is regular. The regular map piv : $C_{X} \rightarrow$ piv $\left(C_{X}\right)$ is an isomorphism. Indeed if $c_{1}, c_{2} \in C_{X}$ are two points such that $u\left(c_{1}\right)=u\left(c_{2}\right)=u$, then both $c_{1}, c_{2}$ will lie in the 6 -space $\mathbf{P}_{u}^{6}$ (see (2.5.b) and (2.3)(ii)); and since $\mathbf{P}_{u}^{6} \subset \hat{F}$ (see (2.3)(ii)) then the line $L=\operatorname{Span}\left(c_{1}, c_{2}\right) \subset \mathbf{P}_{X}^{2}$ will lie in $\hat{F}$. But then the line $L$ will be a component of $C_{X}=\hat{F} \cap \mathbf{P}_{X}^{2}$, which contradicts the general choice of $X$. Since the plane $\mathbf{P}_{X}^{2}=\operatorname{Span} C_{X}$ does not intersect $\hat{\Sigma} \supset$ piv $\left(C_{X}\right)$ then for any $c \in C_{X}$ the set

$$
S_{c}=W_{c} \cap \mathbf{P}_{X}^{10}=X \cap H_{u(c)} \subset X,
$$

where $u(c)=p i v(c)$, is a hyperplane section of $X$. Since different points $c_{1}, c_{2} \in C$ have different pivots $u\left(c_{1}\right), u\left(c_{2}\right) \in \operatorname{piv}\left(C_{X}\right)$ then $S_{c_{1}} \neq S_{c_{2}}$ for $c_{1} \neq c_{2}$. By construction any surface $S_{c}, c \in C_{X}$ is a complete intersection of two hyperplanes in $W_{c}$, say $S_{c}=W_{c} \cap H_{1} \cap H_{2}$. Since the 5-fold $W_{c}$ is singular along the Veronese cone $K_{\hat{u}(c)}$ (see (4.1)), then the surface $S_{c}=W_{c} \cap H_{1} \cap H_{2}$ is singular along the intersection cycle

$$
C_{c}=K_{\hat{u}(c)}=K_{\hat{u}(c)} \cap H_{1} \cap H_{2}=K_{\hat{u}(c)} \cap \mathbf{P}_{X}^{10} .
$$

Assume that $\hat{u}(c) \in C_{c}$. Then $\hat{u}(c) \in H_{1} \cap H_{2}$; and since $X=H_{c} \cap H_{1} \cap H_{2}$ and $H_{c}$ is singular at $\hat{u}(c)$ (see (2.7)(i)) then $X$ will be singular at $\hat{u}(c)$, which contradicts the general choice of $X$. Therefore $C_{c}$ is a codimension 2 linear section of the Veronese cone $K_{\hat{u}(c)}$, which does not contain the vertex $\hat{u}(c)$ of $K_{\hat{u}(c)}$, i.e. $C_{c}$ is a rational normal quartic curve (including the case when $C_{c}$ is a union of two conics intersecting each other at a point). 
(4.4) Lemma. Let $c \in C_{X}$ and let $q(c) \subset \mathbf{P}_{\hat{u}(c)}^{2}$ be the vertex conic of $c$. Then for any $x \in q(c)$ the intersection $q_{x}=Q_{x} \cap S_{c}$ is a conic. If $c \in C_{X}$ is general then for the general $x \in q(c)$ the conic $q_{x}$ is a bisecant to the rational normal quartic $C_{c} \subset \operatorname{Sing} S_{c}$. Moreover for any $c \in C_{X}$ the surface $S_{c}=\cup_{x \in q(c)} q_{x}$.

Proof. Let $c \in C_{X}$, etc. be as above. We shall see first that for any $x \in q(c)$ the quadric $Q_{x} \subset W_{c}$ (see (4.2)) intersects the Veronese cone $K_{\hat{u}(c)}$ along a cone $K_{\hat{u}(c)}^{x} \subset K_{\hat{u}(c)}$ over a conic on the Veronese surface. Indeed the intersection $K_{\hat{u}(c)}^{x}=Q_{x} \cap K_{\hat{u}(c)}=\{w \in \Sigma: x \in$ $\left.\mathbf{P}_{w}^{2} \& \operatorname{dim}\left(\mathbf{P}_{w}^{2} \cap \mathbf{P}_{\hat{u}}^{2}\right) \geq 1\right\}$ coincides with the union of lines $\cup\left\{l_{L}: x \in L \subset \mathbf{P}_{\hat{u}}^{2}\right\}$ on $\Sigma$, see (3.1). Any such $l_{L}$ is a ruling line of the cone $K_{\hat{u}(c)}$. Therefore $K_{\hat{u}(c)}^{x} \subset K_{\hat{u}(c)}$ is a cone; and since the base set $\left\{L \subset \mathbf{P}_{\hat{u}}^{2}: x \in L\right\}$ is a line in $\hat{\mathbf{P}}_{\hat{u}}^{2}$ then the base of $K_{\hat{u}(c)}^{x}$ is a conic on the Veronese surface. By (4.2), for any $x \in q(c)$ the quadric $Q_{x}$ lies in $W_{c}$; and since $X=W_{c} \cap \mathbf{P}_{X}^{10}=W_{c} \cap H_{1} \cap H_{2}$ then the cycle $q_{x}:=Q_{x} \cap \mathbf{P}_{X}^{10}=Q_{x} \cap H_{1} \cap H_{2} \subset W_{c} \cap \mathbf{P}_{X}^{10}=X$ will be a quadric of dimension at least 1. Since $\operatorname{Pic}(X)=\mathbf{Z} . H$, where $H$ is the hyperplane section, then the prime Fano 3 -fold $X=X_{16}$ can't contain quadric surfaces.

Therefore for any $c \in C_{X}$, and any $x \in q(c)$, the cycle $q_{x}$ is a conic; and $q_{x}$ intersects the rational quartic $C_{c} \subset$ Sing $S_{c}$ at the cycle

$$
z_{x}=q_{x} \cap C_{c}=K_{\hat{u}(c)}^{x} \cap H_{1} \cap H_{2} .
$$

By construction, the 4 -fold $W_{c}$ and the rank 3 quadratic cone $K_{\hat{u}(c)}^{x}=Q_{x} \cap K_{\hat{u}(c)}$ depend only on the choice of the point $c \in \hat{F}-\hat{\Omega}$ and the point $x$ on its vertex conic $q(c)$. Therefore for the general codimension 2 linear section $X=W_{c} \cap H_{1} \cap H_{2}$, the cycle $z_{x}=K_{\hat{u}(c)}^{x} \cap H_{1} \cap H_{2}$ will be a general section of $K_{\hat{u}(c)}^{x}$ with two hyperplanes, and then $q_{x} \subset X$ will be a smooth conic on $S_{c}$ bisecant to the rational normal quartic $C_{c}$. For arbitrary $x \in q(c), c \in C_{X}$, the cycle $z_{x}$ is a linear section, of dimension $\leq 1$, of the quadratic cone $K_{\hat{u}(c)}^{x}$. In the extremal case when $\operatorname{dim} z_{x}=1$, the curve $C_{c}$ must be a union of two conics on the Veronese surface such that $z_{x}=q_{x}$ is one of these two conics. This can be possible for at most two conics from the pencil $\left\{q_{x}: x \in q(c)\right\}$. In all the rest possible cases $z_{x}$ will be a zero-cycle of degree 2 .

At the end, the conics $q_{x}, x \in q(c)$ sweep the surface $S_{c}$ out, since $S_{c}=W_{c} \cap H_{1} \cap H_{2}=$ $\cup_{x \in q(c)} Q_{x} \cap H_{1} \cap H_{2}=\cup_{x \in q(c)} q_{x}$, see (4.2). q.e.d.

(4.5) The Fano surface $\mathcal{F}(\mathbf{X})$ and the vertex surface $\mathbf{S}_{\mathbf{X}}$. For the general $X$ the family $\mathcal{F}(X)$ of conics on $X$ is a union of 2 -dimensional components $\mathcal{F}^{1}, \ldots, \mathcal{F}^{n}$, see $\S 4$ in [1]] or $\S 4.2$ in $[\mathrm{IP}]$. We shall see that $n=1$, and $\mathcal{F}(X)=\mathcal{F}^{1}$ is a ruled surface over the dual plane quartic $C_{X}$ of $X$.

(4.5.a) Lemma. The general $X=X_{16}$ does not contain conics of rank 1 .

Proof. For the general $X$, the codimension 9 Schubert cycle $\sigma_{333}\left(\mathbf{P}_{X}^{10}\right)=G\left(2, \mathbf{P}_{X}^{10}\right)$, in $G(3,14)=G\left(2: \mathbf{P}^{13}\right)$, does not intersect the 8-fold $\mathcal{F}_{1}(\Sigma) \subset G(3,14)$ of formal conics of rank 1 on $\Sigma$, see (3.7). q.e.d.

(4.5.b) The vertex map. By (3.5) and (4.5.a), for the general $X$ the map

$$
\text { ver }: \mathcal{F}(X) \rightarrow \mathbf{P}^{5}, q \mapsto \operatorname{ver}(q)=\text { the vertex } x(q) \text { of } q
$$

is regular; and we call this map the vertex map of $\mathcal{F}(X)$. 
(4.5.c) Lemma. The vertex map ver sends the family $\mathcal{F}(X)$ isomorphically onto the irreducible closed set

$$
S_{X}=\cup_{c \in C_{X}} q(c) \subset \mathbf{P}^{5},
$$

swept out by the smooth vertex conics $q(c) \subset \mathbf{P}_{\hat{u}(c)}^{2}$ of the points $c \in C_{X}$, see (3.3).

Proof. Let $x \in S_{X}$. Then $\exists c \in C_{X}$ such that $x \in q(c)$. By (4.4) the cycle $q_{x}=Q_{x} \cap S_{c}$ is a conic on $X$ with vertex $x$. Therefore $S_{X} \subset \operatorname{ver}(\mathcal{F}(X))$.

Next, we shall see that $\operatorname{ver}(\mathcal{F}(X)) \supset S_{X}$. For this, let $q \subset X$ be a conic, and let $x=\operatorname{ver}(q)$. Since $q \subset Q_{x}$ is a codimension 2 linear section of the 3 -fold quadric $Q_{x}$ (see (3.5)), and since any hyperplane section $H_{c}, c \in \mathbf{P}_{X}^{2}$ contains $q \subset X$, then the plane $\mathbf{P}_{X}^{2}$ contains a unique point $c=c(x)$ such that $Q_{x} \subset H_{c}$. Since $H_{c}$ contains a 3-dimensional quadric (the quadric $Q_{x}$ ) then, by (3.3)(i), the point $c$ must lie in $\hat{F}$. Therefore $c \in \mathbf{P}_{X}^{2} \cap \hat{F}=C_{X}$. Moreover, by (3.3)(ii), the vertex $x=x(q)=\operatorname{ver}(q)$ must lie in the vertex conic $q(c) \subset \mathbf{P}_{\hat{u}(c)}^{2}$ of $c$; and since $q(c) \subset S_{X}$ then $x=\operatorname{ver}(q) \in q(c)$ lies in $S_{X}$. Therefore $\operatorname{ver}(\mathcal{F}(X)) \supset S_{X}$. Moreover, by the proof of (4.4), the conic $q_{x}$ is the unique conic on $X$ with vertex $x \in q(c)$. Therefore the regular and surjective vertex map ver $: \mathcal{F}(X) \rightarrow S_{X}$ is injective. q.e.d.

(4.5.d) The vertex surface $\mathbf{S}_{\mathbf{X}}$. By (4.5.a-c), the vertex map ver sends $\mathcal{F}(X)$ isomorphically onto the irreducible closed set

$$
S_{X}=\cup_{c \in C_{X}} q(c) .
$$

Therefore $\mathcal{F}(X)$ is irreducible, and $S_{X} \subset \mathbf{P}^{5}$ is a surface which we call the vertex surface of $X$.

Lemma (4.4) and (4.5.a-d) imply the following

(4.6) Theorem. Let $X=X_{16}$ be general. Then the Fano family $\mathcal{F}(X)$ of conics on $X$ is a ruled surface $p: \mathcal{F}(X) \rightarrow C_{X}$ over the dual plane quartic curve $C_{X}$ of $X$. For the point $c \in C_{X}$, the fiber $f_{c}=p^{-1}(c) \subset \mathcal{F}(X)$ coincides with the pencil $\left\{q_{x}, x \in q(c)\right\}$ of conics $q_{x} \subset X$ defined in (4.4). The vertex map ver $: \mathcal{F}(X) \rightarrow \mathbf{P}^{5}$ sends $\mathcal{F}(X)$ isomorphically onto the vertex surface $S_{X}=\cup_{c \in C_{X}} q(c) \subset \mathbf{P}^{5}$ of $X$. For any point $c \in C_{X}$, the isomorphism

$$
\text { ver }: \mathcal{F}(X) \rightarrow S_{X} \subset \mathbf{P}^{5}
$$

sends the fiber $f_{c} \subset \mathcal{F}(X)$ onto the correlative pivot conic $q(c) \subset \mathbf{P}_{\hat{u}(c)}^{2}$ of $c$.

Proof. By (4.4) and (4.5.c), it only rests to see that the vertex surface $S_{X} \cong \mathcal{F}(X)$ is ruled over $C_{X}$, with fibers - the conics $q(c), c \in C_{X}$. For this it is enough to see that if $c_{1}, c_{2} \in C_{X}$, $c_{1} \neq c_{2}$ then the conics $q\left(c_{1}\right)$ and $q\left(c_{2}\right)$ do not intersect each other.

Suppose that $q\left(c_{1}\right) \cap q\left(c_{2}\right) \neq \emptyset$. Let $x \in q\left(c_{1}\right) \cap q\left(c_{2}\right)$, and let $H_{c_{1}}$ and $H_{c_{2}}$ be the hyperplane sections of $\Sigma$ defined by $c_{1}$ and $c_{2}$. Since $x \in S_{X}$ then, by the proof of (4.4), there exists a unique conic $q=q_{x} \subset X$ with vertex $x$. Let also $Q_{x} \subset \Sigma$ be the 3 -fold quadric with vertex $x$; in particular $q_{x}=Q_{x} \cap \mathbf{P}_{X}^{10}$, ibid. By (3.3)(ii), both $H_{c_{1}}$ and $H_{c_{2}}$ must contain $Q_{x}$ since the vertex $x$ of $Q_{x}$ lies in their pivot conics $q\left(c_{1}\right)$ and $q\left(c_{2}\right)$. But by the proof of (4.5.c), there exists a unique point $c=c(x) \in C_{X}$ such that $Q_{x} \subset H_{c}$. Therefore $c_{1}=c_{2}=c$. q.e.d. 


\section{$\S 5$. Prime Fano 3-folds of degree 16 and stable ruled surfaces of invariant 3 over plane quartics}

(5.1) Lemma: the double projection from a line $\mathbf{l} \subset \mathrm{X}_{\mathbf{1 6}}$ (V. Iskovskikh).

(1) Let $X=X_{16} \subset \mathbf{P}^{10}$ be a smooth prime Fano 3-fold of degree 16, and let $l \in X$ be a line. Then the double projection $\pi_{2 . l}$ from the line $l$, given by the non-complete linear system $\left|\mathcal{O}_{X}(1-2 . l)\right|$, defines a birational isomorphism $\pi=\pi_{2 . l}: X \rightarrow \mathbf{P}_{l}^{3}$, where $\mathbf{P}_{l}^{3}$ is the 3 -dimensional projective space.

There exists a smooth curve $C^{l} \subset \mathbf{P}_{l}^{3}$ of genus 3 and of degree 7 , which lies on a unique cubic surface $S=S_{3}$, such that the inverse birational map $\varphi: \mathbf{P}_{l}^{3} \rightarrow X$ is defined by the non-complete linear system $\left|\mathcal{O}_{\mathbf{P}_{l}^{3}}\left(7-2 C^{l}\right)\right|$, and:

(i) The one-dimensional family $\mathcal{Q}_{l}$ of conics $q \subset X$ which intersect $l$ sweeps out the unique effective divisor $Q=Q_{l}$ from the linear system $\left|\mathcal{O}_{X}(3-7 . l)\right|$.

(ii) The double projection $\pi=\pi_{2 . l}$ can be represented as a product $\pi=\tau \circ \rho \circ \sigma^{-1}$, where $\sigma: X^{\prime} \rightarrow X$ is the blowup of $l \in X, \rho: X \rightarrow X^{+}$is a flop over the projection $\pi_{l}: X \rightarrow X^{\prime \prime}$ from $l$, and $\tau: X^{+} \rightarrow \mathbf{P}_{l}^{3}$ is a blow-down of the proper image $Q^{+} \subset X^{+}$of $Q$ onto the curve $C^{l} \subset \mathbf{P}_{l}^{3}$. The extremal curves $q^{+} \subset X^{+}$contracted by $\tau$ are the strict transforms of the conics $q \in \mathcal{Q}_{l}$.

(iii) The unique cubic surface $S=S_{l} \subset \mathbf{P}_{l}^{3}$ through the curve $C^{l}$, is swept out by the one-dimensional family $\mathcal{S}_{l}$ of conics $s \subset \mathbf{P}_{l}^{3}$, intersecting $C^{l}$ at a 0 -cycle of degree 7 . The proper transform $S^{\prime} \subset X^{\prime}$ of $S$ coincides with the exceptional divisor $\sigma^{-1}(l) \subset X^{\prime}$ of $\sigma$. The strict transforms $s^{\prime} \subset X^{\prime}$ of the conics $s \in \mathcal{S}_{l}$ are the extremal curves of $\sigma$.

(iv) There exists a non-negative integer $e=e(l) \leq 5$ (and if $X$ is general and $l \subset X$ is general then $e(l)=5$ ) such that the flop $\rho: X^{\prime} \rightarrow X^{+}$transforms the proper $\sigma$-preimages $l_{1}^{\prime}, \ldots, l_{e}^{\prime} \subset X^{\prime}$ of the $e$ lines $l_{1}, \ldots, l_{e} \subset X$ which intersect $l$ to the proper $\tau$-preimages $l_{i}^{+}, i=1, . ., e$ of $e$ lines $L_{1}, \ldots, L_{e} \subset \mathbf{P}_{l}^{3}$ of $C^{l}$. If $X$ is general and $l \subset X$ is general, then the lines $L_{1}, \ldots, L_{5}$ are the five 4-secant lines to $C^{l}$.

(2) Let $C \subset \mathbf{P}^{3}$ be a smooth curve of genus 3 and of degree 7 , which lies on a unique cubic surface $S=S_{3}$. Then there exists a smooth prime Fano 3-fold $X$ of degree 16 and a line $l \subset X$, such that (1) takes place for $l \subset X$ and $C^{l}=C \subset \mathbf{P}_{l}^{3}=\mathbf{P}^{3}$.

Proof. See [12].

(5.1.a) Remark. The general curve $C \subset \mathbf{P}^{3}$ of genus 3 and degree 7 evidently lies on a unique and smooth cubic surface $S=S_{3}$. Therefore $S=S_{l}$ and $C=C^{l}$ for some $l \subset X$ as in (5.1)(2). One can represent (non-uniquely) the smooth cubic $S_{3} \subset \mathbf{P}^{3}$ as the blowup $S_{3}=\tilde{\mathbf{P}}_{z_{0}, \ldots, z_{5}}^{2}$ of $\mathbf{P}^{2}$ at 6 points $z_{0}, \ldots, z_{5}$, and the curve $C \subset S_{3}$ - as the proper preimage of an element of the system $\left|\mathcal{O}_{\mathbf{P}^{2}}\left(4-z_{1}-\ldots-z_{5}\right)\right|$. Then the five 4-secant lines of $C$ are the proper preimages $L_{i} \subset S_{3}$ of the conics $C_{i} \subset \mathbf{P}^{2}$ passing through the points $z_{0}, \ldots, \hat{z}_{i}, \ldots, z_{5}, i=1, \ldots, 5$.

Notice that the line $L=L_{0}=$ the blowup of the point $z_{0}$ is the unique line on $S_{3}$ which does not intersect $C$, and $L_{1}, \ldots, L_{5}$ are the five lines on $S_{3}$ which intersect $L$.

(5.1.b) Corollary. The family $\Gamma(X)$ of lines on the general prime Fano 3-fold $X$ is a smooth irreducible curve of genus 17.

Proof. By Th. 4.2.7 in $[\mathrm{IP}]$, for the general $X=X_{16}$ the 1-dimensional family $\Gamma(X)$ of lines on $X$ is smooth. It rests to see that $\Gamma(X)$ is irreducible and of genus 17 . 
Let $\pi=\pi_{2 . l}: X \rightarrow \mathbf{P}_{l}^{3}=\mathbf{P}^{3}$ be the double projection of $X$ from the general line $l \subset X$ as in (5.1). If $m \subset X$ is a line which does not intersect $l$ then its proper image $\pi_{*}(m)$ is evidently a line in $\mathbf{P}^{3}$; and let $\pi_{*}(m)$ intersects $C^{l}$ at $k$ points. Since by (5.1)(1), $\varphi=\pi^{-1}$ is defined by the system $\left|\mathcal{O}_{\mathbf{P}^{3}}\left(7-2 C^{l}\right)\right|$, then the proper image $\varphi_{*}\left(\pi_{*}(m)\right)=m$ will be a curve of degree $=$ 7.deg $m(l)-2 k=7-2 k$; and since $m$ is a line then $k=3$. The same argument in the opposite direction gives that any purely 3 -secant line to $C^{l}$ is the proper $\pi$-image of a line $m \subset X$ which does not intersect $l$.

If $m=l_{i}, i=1, . ., 5$ is one of the five 4 -secant lines of $l$ then, by (5.1)(iv), its proper image $\pi_{*}(m)$ in $\mathbf{P}^{3}$ is one of the five 4 -secant lines $L_{i}$ to $C^{l}$. These $L_{i}$ evidently are 4 -tuple singularities of the curve $\operatorname{Sec}_{3}\left(C^{l}\right)$ of the 3 -secant lines of $C^{l}$. Therefore the map

$$
\pi_{*}: \Gamma(X) \rightarrow \operatorname{Sec}_{3}\left(C^{l}\right)
$$

is the normalization of $\operatorname{Sec}_{3}\left(C^{l}\right)$ at its five 4-tuple points $L_{1}, \ldots, L_{5}$. By (5.1)(2), for the general $X$ the curve $C^{l}$ is a general curve of genus 3 and degree 7 in $\mathbf{P}^{3}$. Therefore, by Proposition 2.4 and Theorem 3.6 in $\mathrm{GP}, \Gamma(X)$ is an irreducible smooth curve of genus 17. q.e.d.

(5.2) Deformations and minimal sections of ruled surfaces (see [LN], [S]). The ruled surface $S=\mathbf{P}(F) \rightarrow C$ over the smooth curve $C$ is stable if the rank 2 vector bundle $F \rightarrow C$ is stable. Since the stability of $F$ does not depend on the twist $F \otimes L$ by a line bundle $L$, the definition is correct.

For the section $C_{o} \subset S$, denote by $\varepsilon\left(C_{o}\right)=C_{o}^{2}=\mathcal{O}_{C_{o}}\left(C_{o}\right) \in \operatorname{Pic}\left(C_{o}\right)=\operatorname{Pic}(C)$ its selfintersection divisor, and let $e\left(C_{o}\right)=\operatorname{deg} \varepsilon\left(C_{o}\right)=\operatorname{deg} C_{o}^{2}$. The integer invariant

$$
e(S):=\min \left\{e\left(C_{o}\right): C_{o} \text { is a section of } S\right\}
$$

is always $\leq g=g(C)$. For a fixed $g$, the ruled surfaces $S$ form two deformation classes - even: with $e(S) \equiv g(\bmod .2)$, and $o d d$ : with $e \equiv g-1(\bmod .2)$. The versal deformations in the even (respectively odd) class are ruled surfaces $S$ with $e(S)=g$ (respectively with $e(S)=g-1$ ). For $g \geq 2$ the general $S$ of any of the two versal types - the even and the odd - is stable; and if $\mathcal{S}[g]$ and $\mathcal{S}[g-1]$ denote respectively their moduli spaces, then $\operatorname{dim} \mathcal{S}[g]=\operatorname{dim} \mathcal{S}[g-1]=6 g-6$. The same as above takes place if the smooth base curve $C$ of genus $g \geq 2$ is fixed, with the only difference that then the moduli spaces $\mathcal{S}_{C}[g]$ and $\mathcal{S}_{C}[g-1]$ of versal even and odd ruled surfaces over $C$ both have dimension $3 g-3$, see [S]. In particular, if $g(C)=3$ then the ruled surfaces of invariant 3 over $C$ are exactly the elements of the even versal class, and their moduli space $\mathcal{S}_{C}[3]$ has dimension 6.

Let $S=\mathbf{P}(F) \rightarrow C$ be a stable ruled surface with invariant $e(S)=e$, and let $C_{o} \subset S$ be the section defined by the extension

$$
0 \rightarrow \xi \rightarrow F \rightarrow \eta \rightarrow 0
$$

for $\eta, \xi \in \operatorname{Pic}(C)$, see Proposition 2.6 in Ch. 5 of [H]. In particular $\varepsilon=\varepsilon\left(C_{o}\right)=\eta-\xi$, with operations in $\operatorname{Pic}(C)$ written additively.

The minimal sections of $S$ are these sections $C_{o}$ of $S$ for which $e\left(C_{o}\right)=\operatorname{deg} \varepsilon\left(C_{o}\right)=e(S)$. Equivalently, the section $C_{o} \subset S=\mathbf{P}(F)$ is minimal iff the subbundle $\xi \subset F$ is of the maximum possible degree, or a maximal subbundle of $F$, see Lemma 2.3 in [LN]. By Lemma 2.2 in [LN] the minimal sections $C_{o}$ of $S$ are defined uniquely by their intersection divisors $\varepsilon\left(C_{o}\right)=C_{o}^{2}$. We shall denote by $\operatorname{Min}(S)$ the family of minimal sections of $S$, and by $C_{\varepsilon} \in \operatorname{Min}(S)$ the minimal section of self-intersection divisor $\varepsilon\left(C_{o}\right)=\varepsilon \in \operatorname{Pic}(C)$. 
Let $g \geq 3$, and $g-1 \leq e=e(S) \leq g$. For the fixed minimal section $C_{\varepsilon} \in \operatorname{Min}(S)$, $\operatorname{deg} \varepsilon=e=e(S)$, let $\left[e_{\varepsilon}\right] \in \mathbf{P}_{\varepsilon}^{g-2+e}=\mathbf{P}\left(\left(H^{o}\left(C, K_{C}+\varepsilon\right)^{*}\right)=\mathbf{P}\left(\operatorname{Ext}^{1}(\eta, \xi)\right)\right.$ be the extensionclass point defined by $C_{\varepsilon}$, and let $\Phi_{\varepsilon}: C \rightarrow \mathbf{P}_{\varepsilon}^{g-2+e}$ be the map defined by the complete linear system $\left|K_{C}+\varepsilon\right|$. By Proposition 2.4 in [LN] there exists a canonical bijection between the set $\operatorname{Min}(S)-\left\{C_{\varepsilon}\right\}$ of minimal sections of $S$ different from $C_{\varepsilon}$ and the $e$-secant spaces $\mathbf{P}^{e-1} \subset \mathbf{P}_{\varepsilon}^{g-2+e}$ to the curve $\Phi_{\varepsilon}(C)$ which pass through $\left[e_{\varepsilon}\right]$.

We shall see below that for $g=3$, the general ruled surface $S \in \mathcal{S}[3]$ is the same as the Fano surface $\mathcal{F}(X)$ of conics on a general prime Fano 3 -fold $X=X_{S}$ of degree 16 defined uniquely by $S$, and the family $\operatorname{Min}(S)$ of minimal sections of $S$ is the same as the curve $\Gamma\left(X_{S}\right)$ of lines on $X_{S}$.

(5.3) Non-abelian Brill-Noether loci of type II (see M3]). Let $F \rightarrow C$ be a rank 2 vector bundle over the smooth curve $C$, let $K=K_{C}$ be the canonical bundle of $C$, and let $\nu$ be a nonnegative integer such that $\nu \equiv \operatorname{deg} F \bmod 2$. The non-abelian Brill-Noether locus of type II, associated to the triple $(C, K, F)$, is the set of equivalence classes of bundles

$$
M_{C}(2, K: n F)=\{E \rightarrow C: \operatorname{rank} E=2, \operatorname{det} E=\operatorname{det} F \otimes K, \operatorname{dim} \operatorname{Hom}(F, E) \geq n\} .
$$

It is proved in $\S 6$ of [M3] that $M_{C}(2, K: n F)$ admits a natural scheme structure as certain Pfaffian locus in the moduli space $M_{C}\left(2\right.$, det $\left.F \otimes K_{C}\right)$ of stable rank 2 vector bundles on $C$ of determinant $\operatorname{det} F \otimes K_{C}$.

(5.4) Lemma (S. Mukai).

(a) Let $C \subset \mathbf{P}^{3}$ be a nonsingular plane quartic curve, and let $F$ be a rank 2 stable vector bundle on $C$ such that the ruled surface $S=\mathbf{P}(F) \rightarrow C$ has invariant $e=e(S)=3$. Then the Brill-Noether locus of type II

$$
X=M_{C}(2, K: 3 F)
$$

is a nonsingular prime Fano 3-fold of degree 16.

(b) Let $C_{\varepsilon}$ be the minimal section of $S$ of self-intersection divisor $\varepsilon$, and let $(\mathbf{5 . 2})(*)$, with $\eta-\xi=\varepsilon$, be the extension defined by $C_{\varepsilon}$. Then the locus

$$
l_{\varepsilon}=\left\{E \in X=M_{C}(2, K: 3 F): h^{o}(E(-\eta)) \geq 2\right\} \subset X
$$

is a line on the Fano 3 -fold $X=X_{16} \subset \mathbf{P}^{10}$.

(c) In the notation of (5.2), the map $\Phi_{\varepsilon}: C \rightarrow \mathbf{P}_{\varepsilon}^{4}$ is an embedding; and the projection $p_{\left[e_{\varepsilon}\right]}: \mathbf{P}_{\varepsilon}^{4} \rightarrow \mathbf{P}_{\varepsilon}^{3}$ from $\left[e_{\varepsilon}\right]$ sends $\Phi_{\varepsilon}(C)$ isomorphically onto a space curve $C_{\varepsilon}^{l}$ of degree 7 .

(d) The curve $C_{\varepsilon}^{l} \subset \mathbf{P}_{\varepsilon}^{3}$ is projectively equivalent to the curve $C^{l} \subset \mathbf{P}_{l}^{3}$ defining the inverse of the double projection $\pi_{2 . l}: X \rightarrow \mathbf{P}_{l}^{3}$, see (5.1)(1).

Proof. See $\S 9$ in $M 3$.

(5.5) The associate Fano 3 -fold $\mathbf{X}_{\mathbf{S}}$ of $\mathbf{S}$. According to Remark 9.2 of [M3], the locus $X_{F}=M_{C}(2, K: 3 F)$ does not depend on the twist $F \rightarrow F \otimes L$ by an invertible sheaf $L$ on $C$. That is, the Fano 3 -fold $X_{S}=X_{F}=M_{C}(2, K: 3 F)$ depends only on the choice of the stable ruled surface $S=\mathbf{P}(F) \rightarrow C$. Therefore a given stable ruled surface $S=\mathbf{P}(F) \in \mathcal{S}_{C}[3]$ defines uniquely its associate Fano 3 -fold $X=X_{S}:=M_{C}(2, K: 3 F)$, which is prime and of degree 16 . 
(5.6) Lemma. Let $F \rightarrow C, S=\mathbf{P}(F) \rightarrow C, X=X_{S}, C_{\varepsilon}$, etc. be as in (5.4)-(5.5), and let $\Gamma\left(X_{S}\right)$ be the curve of lines on $X_{S}$. In addition, we shall assume that $X=X_{S}$ is general (see (5.7) below). Then the map

$$
\psi: \operatorname{Min}(S) \rightarrow \Gamma\left(X_{S}\right), C_{\varepsilon} \rightarrow l_{\varepsilon},
$$

defined in (5.4)(b), is an isomorphism.

Proof. Fix a general minimal section $C_{\varepsilon}$ of $S$, and let $(\mathbf{5 . 2})(*)$ be the extension defined by $C_{\varepsilon}$ as in (5.4)(b). By (5.2) and (5.4)(c), the sections $C_{t} \in \operatorname{Min} \mathbf{P}(F)-\left\{C_{\varepsilon}\right\}$ are in a $(1: 1)$-correspondence with the elements of the family $\operatorname{Sec}_{3}^{2}\left(\Phi_{\varepsilon}(C),\left[e_{\varepsilon}\right]\right)$ of 3 -secant planes $\mathbf{P}_{t}^{2}$ to the curve $\Phi_{\varepsilon}(C) \subset \mathbf{P}_{\varepsilon}^{4}$ which pass through the point $\left[e_{\varepsilon}\right]$. Clearly the projection from $\left[e_{\varepsilon}\right]$

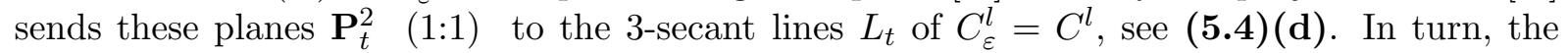
3-secant lines $L_{t}$ of $C_{\varepsilon}^{l}$ correspond, by (5.1.b), to the lines $l_{t} \subset X$ which do not intersect $l=l_{\varepsilon}$. This way, the map $\psi: C_{t} \mapsto l_{t}$ is an isomorphism outside the five lines $l_{1}, \ldots, l_{5}$ which intersect $l=l_{\varepsilon}$, see also (5.1.b).

The same local argument, but applied for other general section $C_{r}$ of $S$ and the line $l_{r} \subset X$ corresponding to $r$, implies that $C_{t} \rightarrow l_{t}$ is an isomorphism also outside the five 4 -secant lines $m_{1}, \ldots, m_{5}$ of the line $m=l_{r}$ on $X$. Since $l$ and $m$ are general, then the sets $\left\{l_{1}, . ., l_{5}\right\}$ and $\left\{m_{1}, . ., m_{5}\right\}$ are disjoint. Therefore $\psi$ is an isomorphism. q.e.d.

(5.7) The associate ruled surface $S^{X}$ of $X$. The inverse to (5.5) is also true: the general Fano 3 -fold $X=X_{16}$ is associate to a unique ruled surface $S$. Indeed, let $C^{l} \subset \mathbf{P}_{l}^{3}$ be the curve defined as in (5.1) by the general line $l \subset X$, and let $H=|K+\varepsilon|$ be the hyperplane system on $C^{l} \subset \mathbf{P}_{l}^{3}$, where $K$ is the canonical class of $C^{l}$. By (5.1)(1)-(2), for the general $l \subset X$, the curve $C^{l}$ is a general curve of degree 7 and of genus 3 in $\mathbf{P}^{3}$. Therefore the complete linear system $|H|$ sends $C^{l}$ isomorphically onto a curve $C \subset \mathbf{P}^{4}$, and $C^{l}$ is a projection of $C$ from a general point $[e] \in \mathbf{P}^{4}$.

By (5.2), the pair $(C,[e])$ represents a minimal section $C_{\varepsilon}$ of the ruled surface $S=\mathbf{P}(F) \in$ $\mathcal{S}[3]$ defined by an extension (5.2) $(*)$ with $\varepsilon=\eta-\xi$. Clearly the surface $S$ is uniquely defined by the pair $(X, l)$. By (5.4), the pair $\left(S, C_{\varepsilon}\right)$ defines uniquely the pair $(X, l), l=l_{\varepsilon} \subset X$, and $X=X_{S}$ does not depend on the choice of the minimal section $C_{\varepsilon}$ of $S$. This, and the isomorphism $\psi: \operatorname{Min}(S) \rightarrow \Gamma\left(X_{S}\right), C_{\varepsilon} \mapsto l_{\varepsilon}$ from (5.6) (more precisely, the surjectivity of $\psi$ ), imply the non-existence of other $S^{\prime}$ with $X=X_{S^{\prime}}$.

We call the unique ruled surface $S=S^{X} \rightarrow C$, such that $X=X_{S}$, the associate ruled surface of $X$; and call its base curve $C=C^{X}$ the associate curve of $X$. By [CG], the description (5.1) of the double projection imply that $C^{X} \cong C^{l}$ (see (5.4)(d)) is the unique curve such that the jacobian $J\left(C^{X}\right)$ is isomorphic, as a principally polarized abelian variety, to the intermediate jacobian $J(X)$ of $X$, see also $\S 9$ in M3.

\section{$\S$. The associate surface $\mathbf{S}^{\mathbf{X}}$ is the Fano surface of $\mathbf{X}$}

(6.1) The double projection from a line $\mathbf{l} \subset \boldsymbol{\Sigma}$. Let $l=l_{L} \subset \Sigma$ be a line with an axis $L=\mathbf{P}\left(U_{2}^{L}\right)$ and a space $\mathbf{P}_{L}^{3}=\mathbf{P}\left(U_{4}^{L}\right)$, see (3.1). By (2.4)(i), for any $u \in L$ the tangent projective space $\mathbf{P}_{u}^{6}$ intersects $\Sigma$ along a cone $K_{u} \subset \mathbf{P}_{u}^{6}$ over the Veronese surface. Let $W_{L}=\cup_{u \in L} \mathbf{P}_{u}^{6}$, and let

$$
Z_{L}=\cup_{u \in L} K_{u}=W_{L} \cap \Sigma .
$$


Then $\mathbf{P}_{L}^{9}:=\operatorname{Span} W_{L}=\operatorname{Span} Z_{L}$ is a 9 -dimensional subspace of $\mathbf{P}^{13}=\operatorname{Span} \Sigma$. We denote by $\check{\mathbf{P}}_{L}^{3}=\left(\mathbf{P}_{L}^{9}\right)^{\perp} \subset \hat{\mathbf{P}}^{13}$ the space of linear equations of $\mathbf{P}_{L}^{9} \subset \mathbf{P}^{13}$.

By its definition, the double projection

$$
\pi_{2 . l}: \Sigma \rightarrow \check{\mathbf{P}}_{L}^{3}
$$

of $\Sigma \subset \mathbf{P}^{13}$ from $l$ is the restriction to $\Sigma$ of the projection $\pi_{2 . l}: \mathbf{P}^{13} \rightarrow \check{\mathbf{P}}_{L}^{3}$ from the subspace $\mathbf{P}_{L}^{9}=\operatorname{Span} Z_{L}$. The codimension 2 cycle $Z_{L} \subset \Sigma$ is the set of points $u \in \Sigma$ where $\pi_{2 . l}$ is non-regular. Let also

$$
Z_{L}^{\prime}=\left\{u \in \Sigma: \operatorname{dim}\left(\mathbf{P}_{u}^{2} \cap \mathbf{P}_{L}^{3}\right) \geq 1\right\}
$$

Since for $u \in \Sigma-Z_{L}^{\prime}$ the lagrangian plane $\mathbf{P}_{u}^{2}$ intersects $\mathbf{P}_{L}^{3}$ at a point, then the map

$$
\varphi_{l}: \Sigma-Z_{L}^{\prime} \rightarrow \mathbf{P}_{L}^{3}, u \mapsto \mathbf{P}_{u}^{2} \cap \mathbf{P}_{L}^{3}
$$

is regular.

(6.2) Lemma. (i) $Z_{L}^{\prime}=Z_{L}$. (ii) There exists a natural identification $\check{\mathbf{P}}_{L}^{3} \cong \mathbf{P}_{L}^{3}$ such that

$$
\varphi_{l}=\left.\pi_{2 . l}\right|_{\Sigma-Z_{L}} .
$$

Proof of (i). Clearly $Z_{L} \subset Z_{L}^{\prime}$; and it rests to see that $Z_{L}^{\prime} \subset Z_{L}$.

Let $u \in Z_{L}^{\prime}$, i.e. $\operatorname{dim}\left(\mathbf{P}_{u}^{2} \cap \mathbf{P}_{L}^{3}\right) \geq 1$. We have to see that $u \in Z_{L}$.

If $\mathbf{P}_{u}^{2} \subset \mathbf{P}_{L}^{3}$ then $u \in l=l_{L}$, since the only lagrangian planes in $\mathbf{P}_{L}^{3}$ are the planes $\mathbf{P}_{w}^{2}, w \in$ $l=l_{L}$, see $(3.1)(\mathrm{ii})$.

If $\operatorname{dim}\left(\mathbf{P}_{u}^{2} \cap \mathbf{P}_{L}^{3}\right)=1$ then the line $M=\mathbf{P}_{u}^{2} \cap \mathbf{P}_{L}^{3}$ will be an isotropic line in $\mathbf{P}_{L}^{3}$, since $M$ lies on the lagrangian plane $\mathbf{P}_{u}^{2}$. Let $Q_{L} \subset G_{L}:=G\left(1: \mathbf{P}_{L}^{3}\right)$ be the set of all isotropic lines in $\mathbf{P}_{L}^{3}$. Clearly $Q_{L}$ contains the hyperplane section $\sigma_{10}(L)=\left\{M \subset \mathbf{P}_{L}^{3}: M \cap L \neq \emptyset\right\}=\{$ the lines which lie in the lagrangian planes $\left.\mathbf{P}_{w}^{2}, w \in L\right\}$. Since the isotropic grassmannian $L G_{2}$ is a hyperplane section of $G(2,6)$ (see (2.1)), then either $Q_{L}=G_{L}$, or $Q_{L}$ will coincide with the hyperplane section $\sigma_{10}(L) \subset G_{L}$. But if $Q_{L}=G_{L}=G(2,4)$, then in $\mathbf{P}_{L}^{3}$ will lie two 3-fold families of lagrangian planes, which is impossible since the only lagrangian planes in $\mathbf{P}_{L}^{3}$ are the planes $\mathbf{P}_{w}^{2}, w \in L$, see (3.1)(ii). Therefore $Q_{L}=\sigma_{10}(L)$. In particular $M \cap L \neq \emptyset$; and since both $M$ and $L$ lie in $\mathbf{P}_{L}^{3}$ then the plane $\operatorname{Span}(M \cup L)$ will be one, say $\mathbf{P}_{v}^{2}$, of the planes $\mathbf{P}_{w}^{2}, w \in l=l_{L}$, see (3.1)(i). Since $\mathbf{P}_{u}^{2}$ intersects $\mathbf{P}_{v}^{2}$ along a line then $u \in K_{v}$, see (2.4)(i). Therefore $Z_{L}^{\prime} \subset \cup_{v \in L} K_{v}=Z_{L}$. q.e.d.

Proof of (ii). By the $S p_{3}$-homogeneity of $L G_{2}$, it is enough to prove (ii) for a particular line $l \subset \Sigma$; and let $l=l_{L}=\operatorname{Span}\left(e_{123}, e_{423}\right), L=\mathbf{P}\left(U_{2}^{L}\right)=\mathbf{P}\left(<e_{2}, e_{3}>\right), U_{4}^{L}=<e_{1}, e_{2}, e_{3}, e_{4}>$, etc. be as in the proof of (3.1)(ii). Then $\mathbf{P}_{L}^{3}=\mathbf{P}\left(U_{4}^{L}\right)=\mathbf{P}^{3}\left(x_{1}: x_{2}: x_{3}: x_{4}\right) \subset \mathbf{P}^{5}=\mathbf{P}\left(V_{6}\right)$, and $\mathbf{P}_{L}^{9}=\operatorname{Span} Z_{L}=\left(x_{156}=x_{256}=x_{356}=x_{456}=0\right)=\left(y_{11}=y_{12}=y_{13}=z=0\right) \subset \mathbf{P}^{13}$. Therefore

$$
\check{\mathbf{P}}_{L}^{3}=\mathbf{P}^{3}\left(x_{156}: x_{256}: x_{356}: x_{456}\right) \cong \mathbf{P}\left(U_{4}^{L} \otimes \wedge^{2} V_{6} / U_{4}^{L}\right) \cong \mathbf{P}_{L}^{3} .
$$

Under this identification, we shall verify first the coincidence $\varphi_{l}=\left.\pi_{2 l}\right|_{\Sigma-Z_{L}}$ over the open subset

$$
\Sigma^{o}:=\Sigma \cap(u=0)=\exp \left(\operatorname{Sym}^{2} \mathbf{C}^{3}\right)=\left\{\left(1: X: \wedge^{2} X: \operatorname{det} X\right):{ }^{t} X=X\right\} .
$$

By (2.1), the lagrangian 3-spaces $U=U_{X} \in \Sigma^{o} \subset G(3,6)^{\circ}$ are parameterized by the symmetric linear maps $X=X_{U}: U_{o} \rightarrow U_{\infty}$, such that $U=<e_{1}+X\left(e_{1}\right), e_{2}+X\left(e_{2}\right), e_{3}+X\left(e_{3}\right)>$. 
For such $U=U_{X}$ and $X=X_{U}$, the Plücker image $p l\left(U_{X}\right)=\mathbf{P}\left(\wedge^{3} U_{X}\right)$ is the same as the point $\exp (X)=\left(1: X: \wedge^{2} X: \operatorname{det} X\right)$, ibid. In other words, for $u \in \Sigma^{o} \subset \mathbf{P}^{13}$ one has: $u=\exp \left(X_{U}\right)$, where $\mathbf{P}(U)=\mathbf{P}_{u}^{2}$ is the lagrangian projective plane of $u$.

Let $u \in \Sigma^{o}, u \notin Z_{L}$, let $\mathbf{P}_{u}^{2}=\mathbf{P}(U)$ be the lagrangian plane of $u$, and let $X=X_{U}$. We shall compute separately $\varphi_{l}(u)$ and $\pi_{2 . l}(u)$.

On the one hand, $\varphi_{l}(u)$ is the intersection point of $\mathbf{P}_{u}^{2}$ and $\mathbf{P}_{L}^{3}$. Since $\mathbf{P}_{u}^{2}=\mathbf{P}(U)=$ $\mathbf{P}\left(<e_{1}+X\left(e_{1}\right), e_{2}+X\left(e_{2}\right), e_{3}+X\left(e_{3}\right)>\right)$, and $\mathbf{P}_{L}^{3}=\mathbf{P}\left(U_{4}^{L}\right)=\mathbf{P}\left(<e_{1}, e_{2}, e_{3}, e_{4}>\right)$, then $U \cap U_{4}^{L}=<\operatorname{det}\left(X_{11}\right) e_{1}+\operatorname{det}\left(X_{12}\right) e_{2}+\operatorname{det}\left(X_{13}\right) e_{3}+\operatorname{det}(X) e_{4}>$, i.e.

$$
\left.\varphi(u)=\left(\operatorname{det} X_{11}: \operatorname{det} X_{12}: \operatorname{det} X_{13}: \operatorname{det} X\right)\right) \in \mathbf{P}_{L}^{3}=\mathbf{P}^{3}\left(x_{1}: x_{2}: x_{3}: x_{4}\right),
$$

where for any $(i, j), X_{i j}$ is the $(i, j)^{t h}$ adjoint matrix of $X$.

On the other hand, as a point of $\Sigma \subset \mathbf{P}^{13}, u=\exp (X)=(1: X: Y: z) \in \Sigma \subset \mathbf{P}^{13}$, where $X=X_{U}, Y=\wedge^{2} X, z=\operatorname{det} X$. Since $\pi_{2 . l}$ is the projection from $\mathbf{P}_{L}^{9}$ onto $\check{\mathbf{P}}_{L}^{3}=$ $\mathbf{P}^{3}\left(x_{156}: x_{256}: x_{356}: x_{456}\right)=\mathbf{P}^{3}\left(y_{11}: y_{12}: y_{13}: z\right)$, then

$$
\pi_{2 . l}(u)=\pi_{2 l}(\exp (X))=\left(\left(\wedge^{2} X\right)_{11}:\left(\wedge^{2} X\right)_{12}:\left(\wedge^{2} X\right)_{13}: \operatorname{det} X\right) \in \mathbf{P}^{3}\left(y_{11}: y_{12}: y_{13}: z\right) .
$$

Since $\left(\wedge^{2} X\right)_{i j}=\operatorname{det} X_{i j}, i, j=1,2,3$, then $\varphi_{l}(u)=\pi_{2 . l}(u)$, under the above identification between $\mathbf{P}^{3}\left(x_{1}: x_{2}: x_{3}: x_{4}\right)=\mathbf{P}_{L}^{3}$ and $\mathbf{P}^{3}\left(y_{11}: y_{12}: y_{13}: z\right)=\check{\mathbf{P}}_{L}^{3}$.

The map $\varphi_{l}$ is evidently surjective onto the 3 -space $\mathbf{P}_{L}^{3}$; and since $\varphi_{l}$ coincides with the projective-linear map $\pi_{2 l}$ over an open subset of $\Sigma$, then $\varphi_{l}$ is a restriction of a projection of $\Sigma \subset \mathbf{P}^{13}$ from a 9 -space $\mathbf{P}_{o}^{9} \subset \mathbf{P}^{13}$. Since $\pi_{2 . l}$ is a projection from the 9 -space $\mathbf{P}_{L}^{9}=\operatorname{Span}\left(Z_{L}\right)$, and since $Z_{L}$ is the non-regular locus of $\varphi_{l}$, then $\mathbf{P}_{o}^{9} \supset \operatorname{Span}\left(Z_{L}\right)=\mathbf{P}_{L}^{9}$. Therefore $\mathbf{P}_{o}^{9}=\mathbf{P}_{L}^{9}$, i.e. $\varphi_{l}=\left.\pi_{2 . l}\right|_{\Sigma-Z_{L}}$. q.e.d.

(6.3) The double projection from a line $1 \subset X$ and the $S_{3} p_{3}$-geometry.

Let $l \subset X=\Sigma \cap \mathbf{P}_{X}^{10}$ be a line, let $L \subset \mathbf{P}^{5}$ be the axis of $l=l_{L}$, and let $\mathbf{P}_{L}^{3} \subset \mathbf{P}^{5}$ be the space of $L$, see (3.1). The double projection $\pi_{2 . l}: X \rightarrow \mathbf{P}_{l}^{3}$, described in (5.1), is also the restriction to $X$ of the double projection $\pi_{2 . l}: \Sigma \rightarrow \mathbf{P}^{3}=\mathbf{P}_{L}^{3}$. By (6.1), $\pi=\pi_{2 . l}: X \rightarrow \mathbf{P}_{L}^{3}$ is non-regular along the subset $z_{l}=Z_{L} \cap \mathbf{P}_{X}^{10}$, and by (5.1), the set $z_{l}=l \cup l_{1} \cup \ldots \cup l_{e}$, where $l_{1}, \ldots, l_{e}$ are the $e=e(l) \leq 5$ lines on $X$ which intersect $l$. By (6.2), for any $u \in X-z_{l}$ the double projection $\pi=\pi_{2 . l}: X \rightarrow \mathbf{P}_{l}^{3}$, described in (5.1), is given by:

$$
\pi: u \mapsto \pi(u)=\mathbf{P}_{u}^{2} \cap \mathbf{P}_{L}^{3} .
$$

This identifies $\mathbf{P}_{L}^{3}$ and $\mathbf{P}_{l}^{3}$; and we shall find the curve $C^{l} \subset \mathbf{P}_{L}^{3}$, see (5.1)(1).

Let $l_{1}, \ldots, l_{e}, e \leq 5$ be the lines on $X$ which intersect $l$; and assume for simplicity that $e=5$, see $(5.1)(\mathbf{1})(\mathbf{i v})$. Let $L_{1}, \ldots, L_{5}$ be the axes of $l_{1}, \ldots, l_{5}$, see (3.1). Let $u_{i}=l \cap l_{i}$, and let $\mathbf{P}_{u_{i}}^{2} \subset \mathbf{P}^{5}$ be the lagrangian plane of $u_{i}$. Since $u_{i} \in l$ then the axis $L$ of $l=l_{L}$ lies in $\mathbf{P}_{u_{i}}^{2}$, and since $u_{i} \in l_{i}$ then $L_{i} \subset \mathbf{P}_{u_{i}}^{2}$. Therefore $L_{i}$ intersects $L$, and let $x_{i}=L \cap L_{i}, i=1, \ldots, 5$. The point $x_{i}=x\left(l+l_{i}\right)$ is the vertex of the conic $q_{i}=l+l_{i}$, i.e. $x_{i} \in S_{X}$; and let $\delta_{L}=\left\{x_{1}, \ldots, x_{5}\right\}$. Since $l_{1}, \ldots, l_{5}$ are all the lines on $X$ which intersect $l$ then

$$
L \cap S_{X}=\delta_{L} .
$$


In the general case $\delta_{L}$ is the intersection 0 -cycle of $L$ and $S_{L}$, see (5.1)(1)(iv). Let

$$
C_{L}=\text { the closure of } S_{X} \cap\left(\mathbf{P}_{L}^{3}-L\right) .
$$

Then $S_{L} \cap \mathbf{P}_{L}^{3}=C_{L} \cup \delta_{L}$; and we shall see that $C_{L}=C^{l}$.

Let first $x \in C_{L} \subset S_{X} \cap \mathbf{P}_{L}^{3}$. Since $x \in S_{X}$, then by (4.5.c) there exists a unique conic $q=q_{x} \subset X$ with vertex $x$; and by $(*)$ the double projection $\pi_{2 . l}$ contracts the conic $q_{x}$ to the point $x=x(q) \in \mathbf{P}_{L}^{3}=\mathbf{P}_{l}^{3}$; in particular $x \in C^{l}$, see (5.1)(1)(ii). Therefore $C_{L} \subset C^{l}$.

The same argument in the inverse direction gives $C^{l} \subset C_{L}$.

Notice that for the general $l$ the curve $C^{l}=C_{L}$ does not intersect the axis $L$ of the line $l=l_{L}$, see (5.1.a). By (5.1)(1)(iv), the five axes $L_{1}, \ldots, L_{5}$ are exactly the five 4 -secant lines to $C^{l}=C_{L}$, see also (5.1.a) and (5.1.b).

(6.4) Corollary. In the notation of (6.3), $C_{L}=C^{l}$.

(6.5) Theorem. Let $X$ be a general prime Fano threefold of degree 16. Then the associate ruled surface $S^{X}$ of $X$ is isomorphic to the Fano surface $\mathcal{F}(X)$ of conics on $X$.

Proof. Let $C^{X}$ and $C_{X}$ be correspondingly the associate and the dual curve of $X$. By (4.6), it is enough to prove that the ruled surface $p^{X}: S^{X} \rightarrow C^{X}$ is isomorphic to the ruled vertex surface $p_{X}: S_{X} \rightarrow C_{X}$ of $X$.

Let $l=l_{L} \subset X$ be a line, and let $C_{\varepsilon} \cong C^{X}$ be the minimal section of $S^{X}$ such that $l=l_{\varepsilon}$, see (5.4), (5.6). Let also $C^{l} \subset \mathbf{P}_{l}^{3}$ be the curve defined by the double projection from $l$ as in (5.1), and let $C_{\varepsilon}^{l} \subset \mathbf{P}_{\varepsilon}^{3}$ be the curve defined in (5.4)(c).

By (5.4)(c)-(5.4)(d) $C^{X} \cong C^{l}$; and by (6.4) $C^{l} \cong C_{L}$. Since both $C^{X}$ and $C_{X}$ are curves of genus 3 , then $C_{L} \subset S_{X}$ is a section of $S_{X}$. Therefore

$$
C^{X} \cong C_{L} \cong C_{X}=: C \text {. }
$$

Let $\operatorname{Min}^{\prime}\left(S_{X}\right)=\left\{C_{L}: l=l_{L} \in \Gamma(X)\right\}$ be the family of all sections $C_{L} \subset S_{X}$. One can see separately that $\operatorname{Min}^{\prime}\left(S_{X}\right)=\operatorname{Min}\left(S_{X}\right)$, but in the proof we will use only the isomorphism $\operatorname{Min}^{\prime}\left(S_{X}\right) \cong \operatorname{Min}\left(S^{X}\right)$. In fact, by (5.4)(c)-(d) and (6.4)

$$
C^{l}=C_{\varepsilon}^{l}=C_{L} \text { in } \mathbf{P}_{l}^{3}=\mathbf{P}_{\varepsilon}^{3}=\mathbf{P}_{L}^{3} ;
$$

and then by (5.6) the maps

$$
\operatorname{Min}^{\prime}\left(S_{X}\right) \stackrel{\xi}{\longrightarrow} \operatorname{Min}\left(S^{X}\right) \stackrel{\psi}{\longrightarrow} \Gamma(X)=: \Gamma, \quad C_{L} \mapsto C_{\varepsilon} \mapsto l_{\varepsilon}
$$

are isomorphisms.

It rests to see that $S^{X} \cong S_{X}$. For this, define the following maps:

$$
\alpha: C \times \Gamma \rightarrow S_{X}, \alpha:(c, l) \mapsto x .(c),
$$

where $l=l_{L}$, and $x .(c):=\left(p_{X}\right)^{-1}(c) \cap C_{L} \in S_{X}$, for $c \in C=C_{X}$; and

$$
\beta: C \times \Gamma \rightarrow S^{X}, \beta:(c, l) \mapsto x \cdot(c),
$$

where $l=l_{\varepsilon}$, and $x \cdot(c):=\left(p^{X}\right)^{-1}(c) \cap C_{\varepsilon} \in S^{X}$, for $c \in C=C^{X}$.

In order to prove that $S_{X} \cong S^{X}$, it is enough to identify the fibers $\alpha^{-1}(\alpha(c, l))$ and $\beta^{-1}(\beta(c, l))$, for any $(c, l) \in C \times \Gamma$. 
Let $(c, l) \in C \times \Gamma$. By the definition of $\alpha$

$$
\alpha^{-1}(\alpha(c, l)) \cong\left\{C_{L_{i}} \subset S_{X}: l_{L_{i}} \subset X \& x .(c) \in C_{L_{i}}\right\},
$$

and one of these sections is $C_{L}, l=l_{L}$. Let $q_{x .(c)}$ be the unique conic on $X$ with vertex $x .(c)$, see (4.5.c) or the proof of (4.4). Since

$$
q_{x .(c)}=Q_{x .(c)} \cap \mathbf{P}_{X}^{10}=\left\{u \in X: x .(c) \in \mathbf{P}_{u}^{2}\right\}
$$

(see e.g. (6.3) or the proof of (4.4)), then the line $l_{i}=l_{L_{i}} \subset X$ passes through $x .(c)$ iff $l_{i} \cap q_{x .(c)} \neq \emptyset$. Therefore

$$
\alpha^{-1}(\alpha(c, l)) \cong\left\{l_{i} \in \Gamma(X): q_{x .(c)} \cap l_{i} \neq \emptyset\right\} .
$$

Clearly, the line $l$ is one of these lines.

By the definition of $\beta$

$$
\beta^{-1}(\beta(c, l)) \cong\left\{C_{t} \subset S^{X}: x \cdot(c) \in C_{t}\right\},
$$

and one of these sections is $C_{\varepsilon}, l=l_{\varepsilon}$. By (5.2) and (5.4)(c) (see also the proof of (5.6)) the minimal sections $C_{t}$ of $S^{X}$, different from $C_{\varepsilon}$, are parameterized by the 3-secant lines $M$ to $C_{\varepsilon}^{l} \subset \mathbf{P}_{\varepsilon}^{3}$. Since $C_{\varepsilon}^{l}=C_{L} \subset \mathbf{P}_{L}^{3}=\mathbf{P}_{\varepsilon}^{3}$, then $C_{t}$ intersects $C_{\varepsilon}$ at the point $x(c)$ iff $x .(c) \in M$. Therefore

$$
\beta^{-1}(\beta(c, l)) \cong\left\{M \in \operatorname{Sec}_{3}\left(C_{L}\right): x .(c) \in M\right\} \cup\left\{C_{\varepsilon}\right\} .
$$

By the proof of (5.1.b) the 3-secant lines $M$ to $C_{L}=C^{l}$ are the proper $\pi$-transforms of the lines $m \subset X$. Moreover, by (5.1)(1)(ii) the points $x=x_{q} \in C^{l}$ are the same as the bloweddown strict transforms $q^{+} \subset X^{+}$of the conics $q \subset X$ which intersect $l$. Therefore there exists a unique conic $q=q_{o} \subset X$ such that $x_{q_{o}}=x$.(c). Since by (6.2)-(6.3)(*) the double projection $\pi=\pi_{2 . l}$ contracts $q_{o}$ to its vertex $x_{q_{o}}=x$. $(c)$ then $q_{o}=q_{x .(c)}$, and

$$
\beta^{-1}(\beta(c, l)) \cong\left\{l_{i} \in \Gamma(X): q_{x .(c)} \cap l_{i} \neq \emptyset\right\} \cong \alpha^{-1}(\alpha(c, l))
$$

q.e.d.

(6.6) Remark. In fact, the coincidence between the fibers of $\alpha$ and $\beta$ implies the isomorphsm between the images $\alpha(C \times \Gamma) \subset S_{X}$ and $\beta(C \times \Gamma) \subset S^{X}$. But $\alpha$ and $\beta$ are certainly surjective since a conic $q \subset X$ always intersects the surface $R_{X} \subset X$ swept out by the lines $l \subset X$. The general conic $q$ on the general $X$ intersects exactly 8 lines on $X$ since $R_{X} \in\left|\mathcal{O}_{X}(4)\right|$, see (6.4) case (v) in [1]. The virtual number $e(q)=8$ of lines intersecting a conic $q \subset X_{16}$ can be computed also by the Mori theory - see $\S(2.5)-(2.6)$ and table (2.8.2) in [1].

On the general ruled surface $S=S^{X} \rightarrow C^{X}=C$ of invariant $e(S)=3$, one can compute the same number $e(x)=8$ of minimal sections through its general point $x$ as follows (see (5.2)): If $x \in S$ is general then the elementary transformation $\operatorname{elm}_{x}: S \rightarrow S_{x}$ sends $S$ to a general $S_{x} \in \mathcal{S}_{C}[2]$. The proper images of the minimal sections of $S$ through $x$ are all the minimal sections of $S_{x}$. $S_{x}$ always has minimal sections, and let $C_{o} \subset S_{x}$ be one of them. Since $S_{x}$ is general, then $\varepsilon_{o}=C_{o}^{2}:=\mathcal{O}_{C_{o}}\left(C_{o}\right) \in \operatorname{Pic}^{2}\left(C_{o}\right)=\operatorname{Pic}^{2}(C)$ is general, and the linear system $\left|K_{C}+\varepsilon_{o}\right|$ sends $C$ isomorphically to a space curve $C_{6}^{3} \subset \mathbf{P}^{3}$ of genus 3 and of degree 6 . The minimal sections of $S_{x}$, different from $C_{o}$, are in a 1:1 correspondence with the bisecant lines to $C_{6}^{3}$ from the (general) extension-class point $\left[e_{\varepsilon_{o}}\right] \in \mathbf{P}^{3}$ defined by the section $C_{o}$. These lines are 7 , since the projection of $C_{6}^{3} \subset \mathbf{P}^{3}$ from a general point in $\mathbf{P}^{3}$ is a plane sextic with 7 double points. 


\section{References}

[CG] H. Clemens, Ph. Griffiths, The intermediate jacobian of the cubic threefold, Ann. of Math. 95:2, 281-356 (1972)

[D] R. Donagi, On the geometry of grassmannians, Duke Math. J. 44:4, 795-837 (1977)

[GP] L. Gruson, Ch. Peskine, Courbes de l'espace projectif: varietes de secantes, in: Enumerative geometry and classical algebraic geometry, Progress in Mathematics, Vol. 24 (1982), p. 1-31, Birkhauser, Boston-Basel-Stuttgart.

[FH] W. Fulton, J. Harris, Representation theory, Springer-Verlag (1991)

[H] R. Hartshorne, Algebraic geometry Springer-Verlag (1977)

[I1] V. A. Iskovskikh, Fano threefolds II, Math. USSR, Izv. 12:3, 469-506 (1978)

[I2] V. A. Iskovskikh, Double projection from a line on Fano threefolds of the first kind, Math. USSR Sbornik 66 No.1, 265-284 (1990)

[IP] V. A. Iskovskikh, Yu. G. Prokhorov, Algebraic geometry V: Fano varieties, Encycl.Math. Sci. 47, 1-245 (1999)

[IR] A. Iliev, K. Ranestad, Geometry of the lagrangian grassmannian with applications to Brill-Nether loci, e-print math.AG/0209169

[K] T. Kimura, The b-functions and holonomy diagrams of irreducible regular prehomogeneous vector spaces, Nagoya Math. J. 85, 1-80 (1982)

[KS] T. Kimura, M. Sato, A classification of irreducible prehomogeneous vector spaces and their relative invariants, Nagoya Math. J. 65, 1-155 (1977)

[LM1] J. M. Landsberg, L. Manivel, The Projective Geometry of Freudenthal's Magic Square, J. of Algebra 239, 477-512 (2001)

[LM2] J. M. Landsberg, L. Manivel, Representation theory and projective geometry, e-print math.AG/0203260

[LN] H. Lange, M. S. Narasimhan, Maximal Subbundles of rank two vector bundles on curves, Math. Ann. 266, 55-72 (1983)

[M1] S. Mukai, Curves, K3 Surfaces and Fano 3-folds of Genus $\leq 10$, Algebraic geometry and commutative algebra in honor of M. Nagata, Kinokuniya, Tokyo, 357-387 (1987)

[M2] S. Mukai, Biregular classification of Fano 3-folds and Fano manifolds of coindex 3, Proc. Natl. Acad. Sci. USA 86, 3000-3002 (1989)

[M3] S. Mukai, Non-Abelian Brill-Noether theory and Fano 3-folds, Sugaku Expositions 49, 1-24 (1997); e-print math.AG/9704015

[M4] S. Mukai, Curves and Symmetric Spaces, Proc. Japan Acad. 68 Ser. A, 7-10 (1992)

[PR] P. Pragacz. J. Ratajski, Pieri type formula for isotropic grassmannians: the operator approach, Manuscripta Math., 79 No. 2, 127-151 (1993)

[S] W. K. Seiler, Deformations of ruled surfaces J. reine angew. Math. 426, 203-219 (1992)

[T] K. Takeuchi, Some birational maps of Fano 3-folds, Compositio Math. 71, 265-283 (1989)

Atanas Iliev

Institute of Mathematics

Bulgarian Academy of Sciences

Acad. G. Bonchev Str., 8

1113 Sofia, Bulgaria

e-mail: ailiev@math.bas.bg 